\title{
Imagined Spatial Transformation of One's Body
}

\author{
Lawrence M. Parsons \\ Massachusetts Institute of Technology
}

\begin{abstract}
This study examined two related phenomena: (a) the judgment of whether a human body part belongs to the left or right half of the body and (b) the imagined spatial transformation of one's body. In three experiments, observers made left-right judgments of a part of a body whose orientation differed from their own by a rotation about one of 13 axes. To do so, they imagined themselves passing to the orientation of the stimulus. Time for (a) left-right judgments and (b) accompanying imagined spatial transformations depended on the extent of the orientation difference (OD) between the observer and stimulus. More important, time for phenomena (a) and (b) depended strongly, and in the same way, on the direction of $O D$. Further results showed that the rate of imagined spatial transformations can vary strongly for different axes and directions of rotation about an axis. These and other results (e.g., Parsons, 1987a) suggest that temporal and kinematic properties of imagined spatial transformations are more object-specific than could be previously assumed.
\end{abstract}

When similar objects are at the same orientation, people can often readily discriminate differences in the composition and spatial arrangement of the objects' features. However, as the objects differ in orientation, the effort needed to discriminate between identical and just similar pairs increases. Searching for and comparing corresponding features of objects at different orientations can overburden spatial working memory (Parsons, 1986b). One very often finds it more efficient to imagine or to produce physical rotation(s) of one object to an orientation like that of the other (e.g., Hinton \& Parsons, 1987; Shepard \& Metzler, 1971). This latter fact has been exploited to study both the internal representation of shape (Corballis, Zbrodoff, Shetzer, \& Butler, 1978; Hinton \& Parsons, 1981) and imagined spatial transformations (Bundeson, Larsen, \& Farrell, 1981; Just \& Carpenter, 1985; Metzler \& Shepard, 1974; Parsons, 1983a, 1983b, 1986a, 1987a, 1987b, in press).

This article is based in part on a doctoral dissertation for the Department of Psychology at the University of California, San Diego. It was presented in part at the Fifth Annual Conference of Cognitive Science Society, and in part at the 1985 Annual Meeting of the Eastern Psychological Association.

The research reported in this article was supported by National Science Foundation Grant BNS 79-24062 to James L. McClelland; Contract N00014-79-C-0323, NR 667-437 with Personnel and Training Research Programs of the Office of Naval Research; a grant from A. P. Sloan Foundation Program in Cognitive Science to Massachusetts Institute of Technology Center for Cognitive Science; and National Research Service Award Fellowship F32 HD6605-02 from National Institute of Health.

Many thanks to Charles Collyer, James Enns, Roger Shepard, and Barbara Tversky for helpful comments on earlier drafts of this manuscript; to Laurie Carman and Ray Nagey for help with conducting experiments; and to Stuart Hacker and Jeni Yamada for drawing figures.

Correspondence concerning this article should be addressed to Lawrence M. Parsons, Department of Brain and Cognitive Sciences, E10020, Massachusetts Institute of Technology, 77 Massachusetts Avenue, Cambridge, Massachusetts 02139.

\section{Properties of Imagined Spatial Transformations}

The time to imagine an object's reorientation often increases with angle of orientation difference (e.g., Cooper, 1975; Cooper \& Shepard, 1973; Metzler, 1973; Parsons, 1987a). This relation between reaction time (RT) and orientation holds for orientation differences about either the line of sight or vertical axes. Furthermore, the rate of imagined reorientation can vary by more than an order of magnitude depending on the object's complexity or familiarity (e.g., Cooper, 1975; Kaushall \& Parsons, 1981; Parsons, 1983b, 1987a; Shepard \& Hurwich, 1984; Shepard \& Metzler, 1971). Overall, these and related results (e.g., Cooper, 1976; Pinker, 1980) are taken to imply that imagined spatial transformations produce an approximately continuous series of intermediate internal representations of a shape that correspond to its intermediate physical orientations. Such results are also thought to imply that objects are probably internally represented in three dimensions, rather than in two dimensions of projected three-dimensional information (as in a literally "pictorial" representation).

\section{Limits of Previous Imagined Spatial Transformation Studies}

These conclusions are based on research using letters, numbers, or abstract two- and three-dimensional shapes. Furthermore, two planes (or axes) of rotation were most efficient to correct for the difference in orientation between the standard and comparison objects. Most studies used orientation differences (ODs) in one or two planes (picture or depth), and trials were often blocked by the plane of OD. Such experimental designs fail to reflect an important aspect of human spatial transformations. Perceptual, imaginal, or motor systems are capable of interpolating, recognizing, representing, or effecting the efficient displacement of an object from one orientation to any other, with apparently little deliberation. An unsolved problem is the nature of the procedures and economies that allow us to select from among the indefinitely many paths an object can 
traverse. To investigate this problem, researches have begun to study imagined spatial transformations of objects and are attempting to develop conditions under which imagined paths can be compared to those produced by models based on different kinds of geometrical procedure (Just \& Carpenter, 1985; Parsons, 1983a, 1983b, 1986a, 1987a, 1987b, in press).

In the studies reported in this article, the number and variety of orientation differences between the internally represented standard and externally presented comparison objects are relatively unconstrained by experimental design. This affords some examinations of subjects' abilities and preferences for selecting planes or axes for imagined reorientations. These studies attempt to provide evidence discriminating among classes of spatial transformation procedures that differ with respect to the efficiency of their reorientation paths.

The stimuli used in the present experiments are photographs or line drawings of a natural or biological object (the human body), part of which (an arm) may be spatially transformed (outstretched) relative to the whole. In contrast with findings from the initial studies that used human body parts as stimuli (Cooper \& Shepard, 1975), recent findings suggest that properties of the imagined spatial transformation of these stimuli may differ from that of other objects, such as letters, numbers, and abstract, unfamiliar, two- and three-dimensional shapes (Parsons, 1983b, 1987a, 1987b, in press; Sekiyama, 1982, 1983). Information about such possibly different spatial transformations and the accompanying internal representations should be useful in understanding some fundamental processes of spatial cognition.

\section{Geometrical Basis of an Object's Reorientation}

There are infinitely many paths for passing an object from one orientation to another, and a path can be produced by more than one spatial transformation procedure (cf. Parsons, 1986a). To illustrate some of the properties of this geometrical problem (see Figure 1), I will discuss three basic approaches (although there are many possible procedures: see Appendix). Procedures 2 and 3 are examples of the class of procedures that produce paths of overall relatively efficient length; Procedure 1 is an example of the class of overall relatively inefficient procedures.

1. Rotations-by-dimensions: a "decomposition" procedure producing a sequence of rotations about a different axis (e.g., a principal axis of the object or environment) for each dimension by which they differ in orientation.

2. Spin-precession: rotation about an instantaneously changing axis produced by simultaneous rotations about two orthogonal axes (e.g., a principal axis of the object and an axis fixed in the environment, as in a spinning top or celestial body).

3. Shortest path: rotation about an axis (unique for each orientation difference) to simultaneously correct for all differences in orientation while absolutely minimizing the degrees of rotation.

Different spatial transformations have different strengths and weaknesses (cf. Parsons, 1986a). For example, the shortest path for the orientation difference in Figure $1 \mathrm{c}$ is not obvious. This is because the axis of rotation is not coincident with one of the principal axes of the object (the body). For imagining the reorientation of one's body, the paths for rotations-by-dimensions and spin-precession procedures may in general be more obvious, although they will usually be longer by varying amounts.

Here, the focus is on a procedure's (total) angle of rotation, because this has a monotonic, curvilinear relation to reaction time, our usual experimental measure. Each of these three procedures (or some variant) uses the same angle of rotation and path when the orientation difference is due to rotation about a principal axis of the object or environment. Most previous work, which used this kind of orientation difference, could not discriminate among different reorientation procedures. (The exceptions are Just \& Carpenter, 1985; Parsons, 1983b; and Cooper \& Shepard, 1975, although the last authors did not recognize this: see Parsons, 1987a.) In the series of experiments reported here, an attempt is made to assess whether people imagine spatial transformations such that the total extent of rotation is relatively efficient (like shortest path and spin precession) or inefficient (like rotations by dimensions).

\section{Experiments 1-3: Left-Right Judgments of an Outstretched Arm of a Body in Observer's Frontoparallel Plane}

With these issues in mind, I began investigating left-right judgments of parts of the body at many orientations. Pilot subjects viewed the front or back of a human body in the picture (or frontoparallel) plane with an arm outstretched, and pressed a left-hand button if the body's left arm was outstretched and a right-hand button if its right arm was outstretched. The RTs and introspections suggested that these judgments were made by using a method analogous to that used to make left-right judgments of hands and feet (Cooper \& Shepard, 1975; Parsons, 1987a). Apparently, people imagined reorientations of their body from an upright orientation to the orientation of the stimulus to compare the stimulus with their own body. (See related work on the use of the body as an "analogy" for the shape of abstract objects: Parsons, 1986c; Sayeki, 1981; see also the work on the internal representation of the body by Parsons \& Shimojo, in press.)

Experiments 1 and 2 formally demonstrate these findings on left-right judgments by using 13 axes of rotation to create orientation differences between the subject and stimulus. Experiment 3 provides finer analytic information. It uses the paradigm in Experiment 1 to observe discrimination functions for planes of orientation difference that are more representative of the set of possible orientations of a body.

\section{Method}

Subjects. A total of 10 right-handed University of California at San Diego (UCSD) undergraduates who had not been in any related experiments participated for credit in a course in psychology.

Stimuli. Line drawings of the front and back of the body (Figure 1) were presented at 12 picture plane orientations: upright, upside-down, and $30^{\circ}, 60^{\circ}, 90^{\circ}, 120^{\circ}$, and $150^{\circ}$ from upright in clockwise and counterclockwise directions. Stimuli subtended $2.5^{\circ}-5^{\circ}$ of visual angle when displayed in a Gerbrands tachistoscope.

Design. Subjects performed eight blocks of 48 trials each. Every block contained each stimulus at each orientation in a different random order. The first two were practice blocks. Half of the subjects saw one order of test blocks, and the other half saw the reverse order. Trials on 


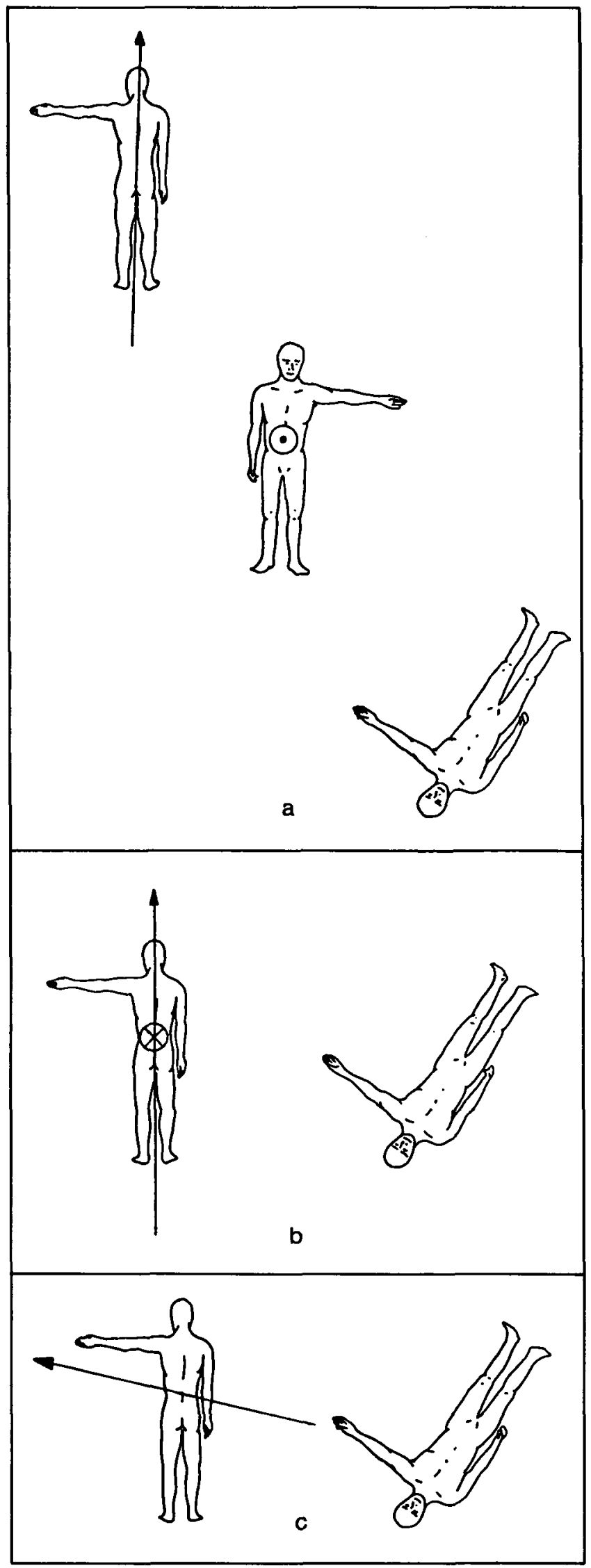

which errors were made were repeated later in a block until performed correctly.

Procedure. Subjects sat before a tachistoscope with their index fingers on a two-key microswitch. They pressed the left button for a stimulus with a left arm outstretched and the right button for a stimulus with right arm outstretched. They were to respond as rapidly and accurately as possible and were not to make head or hand movements. They were given no instruction about how to make their judgments. A trial started with the presentation of a black fixation point on a white background for $2 \mathrm{~s}$. A stimulus was then presented until a response was made. An electronic timer recorded RT (within $I \mathrm{~ms}$ ) and accuracy of response. At the conclusion of the experiment, subjects described in writing their method of performing the task.

\section{Results}

Analyses use RTs of correct responses only. Error rate was less than $2 \%$ on average, and was correlated with RT $(r=.82)$, $F(1,22)=45.76, p<.0001$, for the means in Figure 2 and errors).

An analysis of variance (ANOVA) of RTs to both stimuli was performed with 12 orientation differences (ODs), stimulus type, and stimulus (or response) handedness. Reaction times were longer to fronts than backs, $F(1,9)=9.08, p<.05$, for greater ODs, $F(11,99)=16.70, p<.001$, and effect of $O D$ on RT was different for backs and fronts, $F(11,99)=7.87, p<$ .001 . The RT-OD function for backs had a lower intercept and steeper slope than that for fronts. Linear regressions of OD on RT means for backs and for fronts were reliable, $F(1,5)=14.47$, $p<.05$, and $F(1,5)=9.80, p<.05$. The intercepts of best fit regression lines for these two stimuli were reliably different twotailed $t$ test, $t(5)=4.65, p<.01$. The difference between slopes of best fit regression lines for backs and for fronts was only marginally reliable $(p<.07)$. Linear regression of RT means on OD with three different models of orientation difference (see the predictions in Table Al in the Appendix) showed the following fits. Assuming that subjects used a rotations-by-dimensions procedure, $38 \%$ of the variance was accounted for, $53 \%$ or $50 \%$ of the variance was accounted for by assuming that subjects used a shortest-path or spin-precession procedure, respectively. (These values are reliable to .001 ; the fits assume that the rate and initiation time of imagined spatial transformations are independent of the plane of orientation difference. See later discussions of this issue.)

\section{Discussion}

Model of the left-right judgment of body parts. When leftright, top-bottom, and front-back aspects of the stimulus were

Figure 1. Illustration of three procedures for reorienting an object. ([a] Rotations-by-dimensions path uses a sequence of two rotations: $180^{\circ}$ about the body's major principal axis, then $150^{\circ}$ about its front-back axis. [b] Spin-precession path uses a simultaneous rotation about the body's long axis and about the environmentally fixed axis shown (perpendicular to this page). The effective axis changes instantaneously throughout reorientation, whereas the body's long axis stays in the plane of this page; in this case, a total of $234^{\circ}$ of rotation are required. [c] Shortest path uses a $180^{\circ}$ rotation about the axis shown. The body's long axis swings out of the plane of this page.) 


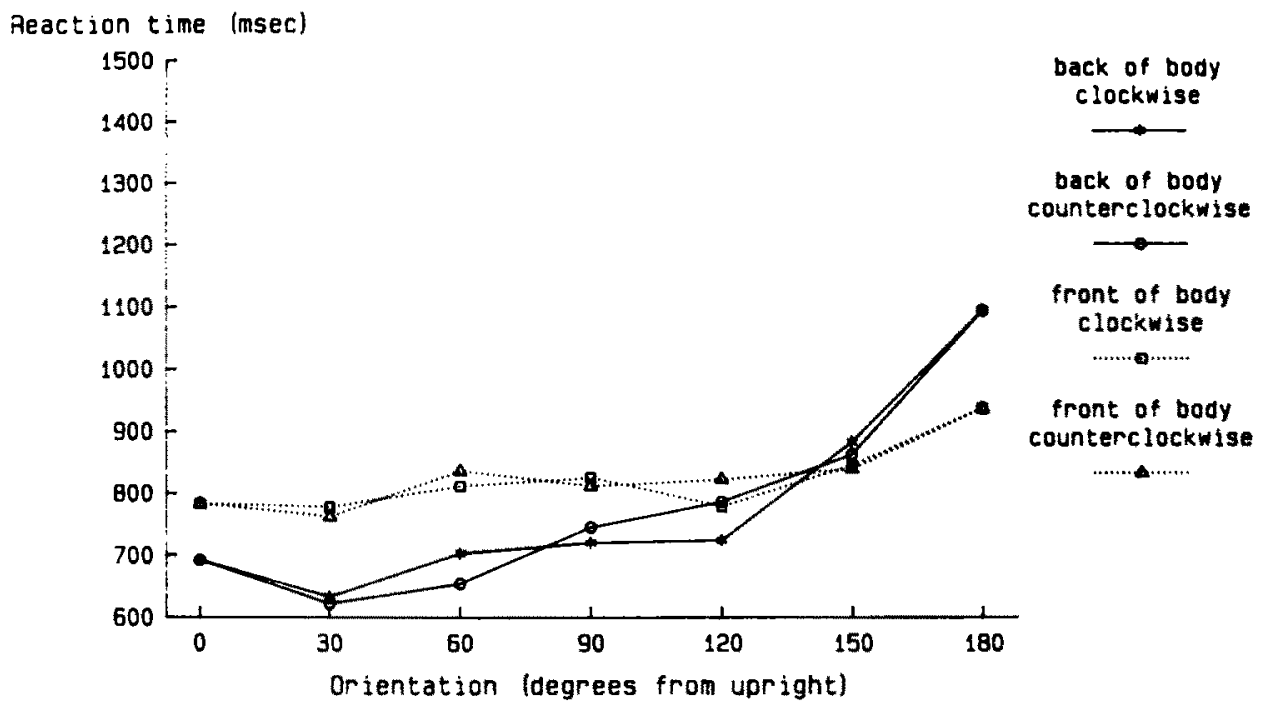

Figure 2. Mean RT as a function of the clockwise and counterclockwise picture plane orientation of the back and front of the body. (Note that this figure is plotted at half the scale of Figures 4-16, with a 600 to 1500 ms RT range rather than 400 to $2200 \mathrm{~ms}$.)

aligned with those of the subjects, subjects reported that it was obvious which arm was outstretched. Accordingly, when the back of the body in the picture plane was at the $0^{\circ} \mathrm{OD}$, subjects produced their shortest RTs. When the stimulus was at other orientations, subjects reported imagining a representation of their own bodies at an upright orientation passing to the orientation of the stimulus for comparison. This contrasts with discrimination of correct from mirror-image letters and numbers (Cooper \& Shepard, 1973; Hinton \& Parsons, 1981), when subjects typically imagine spatial transformations of the stimulus to a standard orientation (upright).' It may be more efficient in this case to imagine a spatial transformation of an internal representation (of one's body) to compare it with an external stimulus than to imagine the rotation of the stimulus and to maintain and compare two internal representations.

It is possible that to establish congruence of the stimulus and an internal representation of their body, subjects imagined a spatial transformation of their arms only, and not their whole bodies. However, no subject reported using this strategy. Further work is necessary to separate possible variation in imagined spatial transformations of the body's parts from variation in imagined spatial transformations of the whole body. Paradigms such as that in Part B of Experiment 2 should be useful for this purpose.

Reaction times. Overall mean RT for this judgment is comparable to that for other familiar stimuli, such as letters, numbers, and well-studied abstract two-dimensional shapes. By contrast, mean RT for left-right judgments of other parts of the body (hands and feet), varies from 700 to $2000 \mathrm{~ms}$, depending on the direction of the orientation difference (Parsons, 1987a). Furthermore, as with discrimination between identical and mirror-image pairs of other types of stimuli, RT depended on the OD between the standard and comparison objects (i.e., the orientation difference between the stimulus and the subject). However, because OD here was about one of many different axes, the interpretation of observed RT-OD functions depends on various assumptions and/or on independently observed information.

The curvilinear function for the back of the body is a more extensive form of the discrimination RT-OD function observed for misoriented letters, numbers, or abstract shapes near some standard orientation (e.g., Cooper \& Shepard, 1973; Kaushall \& Parsons, 1981). In such cases, comparison objects differed from the standard in a single principal plane of the object. Very similar functions are observed for left-right judgments of the back of the hand and top of the foot presented at orientations that apparently differ from the internally represented standard in a single principal plane of the object (Parsons, 1983b, 1987a). Reaction times for the back of the body are consistent with use of rotations-by-dimensions, spin-precession, or shortest-path procedures, because each would produce the same angle and path between the orientation of the subject and stimulus (see Table Al in Appendix).

Reaction times for the front of the body increase slightly with increasing ODs. The use of spin-precession paths would predict such a slightly sloped function, assuming the same rates and initiation times for different planes of spatial transformation. However, the slight slope is also consistent with use of the shortest-path procedure, if, across the range of $O D$, associated rates gradually decrease and/or initiation times gradually increase. Reaction times are probably not consistent with use of the rotations-by-dimensions procedure. To be consistent with the observed gradual slope, rates would have to increase considerably and/or initiation times would have to decrease considerably,

\footnotetext{
${ }^{1}$ Ratcliff (1979) used a task that was simpler but related to the leftright judgment task in the present experiment. He studied the spatial skills of adults with left, right, and bilateral brain lesions. In his analysis, he assumed that subjects imagined rotating the stimulus to upright. The evidence reported here suggests this is incorrect.
} 
across the range of ODs. Linear regressions of RT means on $\mathrm{OD}$, for both front and back of the body, show a worse fit for the rotations-by-dimensions procedure $\left(r^{2}=.38\right)$ than for the other two procedures or models. The shortest-path and spinprecession procedures fit equally well (an $r^{2}$ of about .50).

\section{Experiment 2: Effect of Spatial Stimulus-Response Compatibility and Ambiguous Perspective of Stimuli on Left-Right Judgment}

The findings in this control experiment confirm that RT patterns in Experiment $I$ do not result from either spatial stimulus-response compatibility or the ambiguous perspective of stimuli.

Parts A and B examine the effect of the spatial relation of features of the stimulus and button press response. There is a compatible spatial relation of features when the outstretched left arm of an upright body (in picture plane), seen from the back, points to the observer's left. There is an incompatible spatial relation when the left arm of an upside-down body seen from the back points to the observer's right. Reaction times could be shorter when stimulus-response compatibility is present. The stimulus could direct attention toward the side of the body involved in making the button-press response. Differences in this spatial compatibility of features of stimulus and response may influence RT-OD functions in Experiment 1. This possibility is investigated in two ways. Part A uses a response mode less directly related to left and right spatial coordinates than that of Experiment 1 , but it is otherwise an exact replication. Subjects in Part A respond vocally (saying "left" or "right") rather than pressing a button with the left or right hand.

Part B examines the effect on performance in Experiment 1 when there is an opposite spatial relation between (a) direction the stimulus arm is pointed and (b) side of a subject's body. If spatial stimulus-response compatibility influenced performance in Experiment 1, then subjects should perform differently in a task that has reversed the spatial relations of those features. Subjects in Part B make left-right judgments of stimuli identical to those used in Experiment 1 in all but one respect: The stimulus arm is pointed contralaterally, across its midline (Figure 3).

Part $C$ investigates how performance in Experiment 1 is influenced by perspective information in stimuli. In Experiment 1, there was an ambiguous spatial relation between stimulus and observer with respect to the frame of reference of the environment. Subjects could have seen a stimulus as being viewed from more than one perspective (e.g., looking down at, rather than across at, a stimulus). This may have affected the results. Subjects should be less likely to use such a strategy when the relation between orientation of their body and orientation of the stimulus (with respect to environment) is unambiguous. In Part $\mathrm{C}$, the stimulus body is embedded within an unchanging scene whose frame of reference matches that of the environment (Figure 3).

\section{Method}

Subjects. A total of 18 UCSD undergraduates who had not been in any similar experiments participated for credit in a psychology course. Of those, 6 randomly selected individuals were assigned to each part.
Stimuli, design, and procedure. Stimuli in Part A were those in Experiment 1 . Those for Parts B and C are shown in Figure 3. Stimuli were presented in 12 picture plane orientations. Design of each part exactly replicated Experiment 1 in all but one respect: Subjects performed four blocks of 48 trials each (the first two were practice). Subjects in Part A were to say "left" if left arm of stimulus was raised and "right" if a right arm was raised. All other aspects of procedure in Parts A, and all of Parts $B$ and $C$ procedures were identical to those in Experiment 1.

\section{Results and Discussion}

There were no major differences between performance in the control experiment and performance in Experiment 1. As in Experiment 1, the error rate for each part was less than 3\% and was correlated with RT, $r=.89, F(1,12)=47.94, p<.0001$; $r=.83, F(1,12)=27.44, p<.001 ; r=.88, F(1,12)=41.63$, $p<.0001$. Introspective reports in each part were identical to those in Experiment 1. The ANOVAs of RTs in each part showed no effects of clockwise and counterclockwise stimulus orientations.

An ANOVA of RTs in each part to fronts and backs was conducted with 12 ODs $\left(0^{\circ}-330^{\circ}\right)$, stimulus type, and stimulus handedness. Reaction times were very similar to those in Experiment 1. In Part A, RTs were longer for greater ODs up to $180^{\circ}$, $F(11,55)=11.43, p<.001$, and the effect of OD on RT was different for backs and fronts, $F(11,55)=9.06, p<.001$. In Part B, RTs were longer (a) to fronts than to backs, $F(1,5)=$ $8.37, p<.05$; (b) for greater ODs, $F(11,55)=5.22, p<.001$; and (c) the effect of OD on RT was different for fronts and backs, $F(11,55)=3.70, p<.001$. In Part C, RTs were longer for greater ODs, $F(11,55)=11.75, p<.001$, and the effect of OD on RT was different for backs and fronts, $F(11,55)=8.25, p<.001$.

The RT-OD function in each part for backs had a steeper
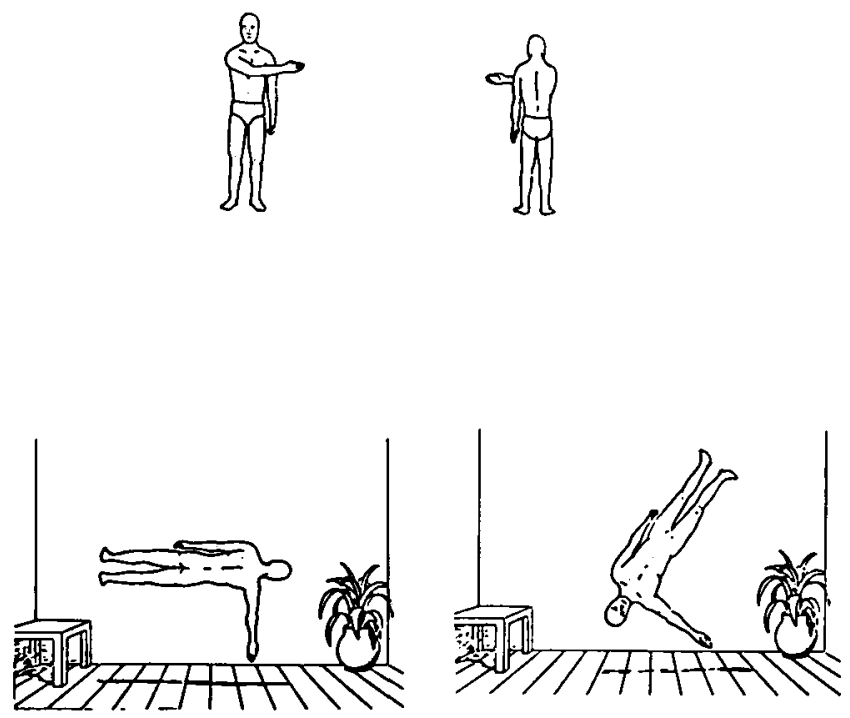

Figure 3. In the upper panel, two of four stimuli in Part B of Experiment 2: the back and front of the body with contralaterally outstretched right arm at orientation difference (OD) of $0^{\circ}$; in the lower panel, two of four stimuli in Part $\mathrm{C}$ of Experiment 2: the back and front of the body with outstretched right arm at $\mathrm{OD}$ of $0^{\circ}$ and shown embedded in an unchanging scene. 
slope and lower intercept than fronts. There were no reliable differences between the slopes and intercepts of linear regression equations of RTs observed in Parts B and $C$ and those observed in Experiment 1 and Part A (two-tailed $t$ test, $p>.05$ ). The intercept of the linear regression equation for fronts is reliably different from that in Experiment $1, t(1,5)=5.42, p<.05$.

In each part, the linear regression of RT on OD for backs of bodies was reliable, $F(1,5)=22.80, p<.01 ; F(1,5)=18.58$, $p<.01 ; F(1,5)=28.85, p<.01$; however, that for fronts of bodies was reliable for RTs in Part C only, $F(1,5)=10.58, p<$ .05 . The intercept of the regression equation for backs is reliably shorter than that for fronts in each part, $t(1,5)=10.44, p<$ $.001 ; t(1,5)=8.11, p<.001 ; t(1,5)=9.24, p<.001$. The slope of the function for backs is reliably steeper than that for fronts in Part $C, t(1,5)=6.27, p<.01$.

\section{Experiment 3: Left-Right Judgments of Outstretched Arm of a Body at a Large, Representative Set of Orientations}

This experiment uses the paradigm of Experiment 1 more analytically to observe discrimination functions for planes of orientation differences created by rotations about a single axis. The set of axes used is much more representative of the set of all possible orientations (see Figures 4-16). The stimuli are color photographs of a human-like doll (with one arm outstretched) in 288 orientations resulting from rotations (in $30^{\circ}$ intervals) about 13 axes.

\section{Method}

Subjects. A total of 13 righthanded Massachusetts Institute of Technology (MIT) undergraduates who had not been in any related experiments participated for $\$ 5$ an hour.

Stimuli. Color photographic slides of a clothed human-like doll were illuminated by a Mast slide projector (with attached tachistoscopic shutter). The standard orientation of the doll (configured as in Figure 1) was upright, with its back to camera and its frontal plane parallel to camera's picture plane. Other orientations were created by passing each of 13 axes through the center of mass of the doll at the standard orientation. The doll was rotated about each axis $180^{\circ}$, and $30^{\circ}, 60^{\circ}, 90^{\circ}, 120^{\circ}$, and $150^{\circ}$ clockwise and counterclockwise. A Cartesian frame of reference was applied to the doll at the standard orientation, so that the $X$ axis was aligned along the ipsilaterally outstretched left arm, the $Y$ axis extended toward the head, and the $Z$ axis extended in the direction that the nose and the camera pointed. The 13 axes are described by unit vectors (Figures 4-16) from the origin (center of mass) of this frame of reference. At each of the resulting 144 orientations, there was one stimulus with left arm outstretched and another with right arm outstretched. The doll (in dark clothes) was shown airborne against a white background; the axis of rotation was not visible. The outstretched arm was bare, and its position was adjusted slightly when it would otherwise be obscured (by the rest of the doll) in the camera's view.

Design and procedure. The 288 stimuli were presented in a random order unique for each block and subject. Each subject performed three blocks (the first was a practice block) in two sessions. Trials on which errors were made were repeated later in a block until performed correctly. The procedure is identical to that in Experiment 1 except that a PDP 11/03 microcomputer controlled the experiment.

\section{Results}

Error rate was less than $3 \%$ on the average and correlated with RT, $r=.78, F(1,142)=223.55, p<.0001$. Analyses used RTs of correct responses only.

Reaction time depended on how much the stimulus orientation differed from that of the subject. There was a slight slope near the upright orientation with an increasing slope for ODs greater than $90^{\circ}$, which may have been caused by subjects' extraexperimental familiarity with upright bodies. In addition, it may partly have been a practice effect because many stimuli showed the body near the upright orientation.

Reaction time also depended on the axis used to create the orientation difference between the stimulus and subjects. An ANOVA of RTs was conducted with 11 ODs, stimulus (or response) handedness, and axis. Reaction time depended on axis, $F(12,144)=20.82, p<.0001$, and orientation, $F(10,120)=$ $99.39, p<.0001$. The effect of OD on RT was different for different axes, $F(120,1440)=10.07, p<.0001$. The effect of axis on RT was different for stimulus (or response) handedness, $F(12,144)=1.90, p<.05$. The effect of OD on RT depended on axis and stimulus (or response) handedness, $F(120,1440)=$ $2.20, p<.0001$.

Effects of stimulus (or response) handedness on RT appear to be related to changes in properties of subjects' imagined spatial transformation in response to whether the left or right stimulus arm is outstretched (see Experiment 4).

Linear regression of RT means on shortest-path OD for each axis was reliable but accounted for only .41 of the variance, $r=$ $.646, F(1,141)=100.98, p<.0001$. This is because RTs strongly depended both on which plane the OD was in and on the clockwise or counterclockwise direction in that plane. Slopes of best fit linear regressions of RT on OD for individual planes of orientation varied by a factor of 2.5 (see Figures 4-16).

The RT-OD functions for various axes are different for clockwise and counterclockwise ODs, and they reflect a tendency for RT to be longer for stimuli showing the top of body pointing away from observer and (especially) below the horizontal plane and away from the observer.

Because RT depended on the plane of OD and clockwise and counterclockwise direction in the plane of OD, models based only on OD are not very predictive. The rotations-by-dimensions procedure is again less consistent with the evidence than the spin-precession and shortest-path procedures. On the basis of a linear regression of RT on OD, a rotations-by-dimensions procedure would account for $26 \%$ of the variance, whereas the spin-precession and shortest-path procedures would account for $39 \%$ and $41 \%$ of the variance (all of which are reliable to $p<.001)$.

\section{Discussion}

Time for left-right judgments depends on direction of orientation difference. These results show that the direction, as well as the extent of the OD between stimulus and observer, greatly influences the time to make a left-right judgment of a part of a misoriented body. Slopes for RT-OD functions for different planes of orientation varied by a factor of 2.5 . The slightest 


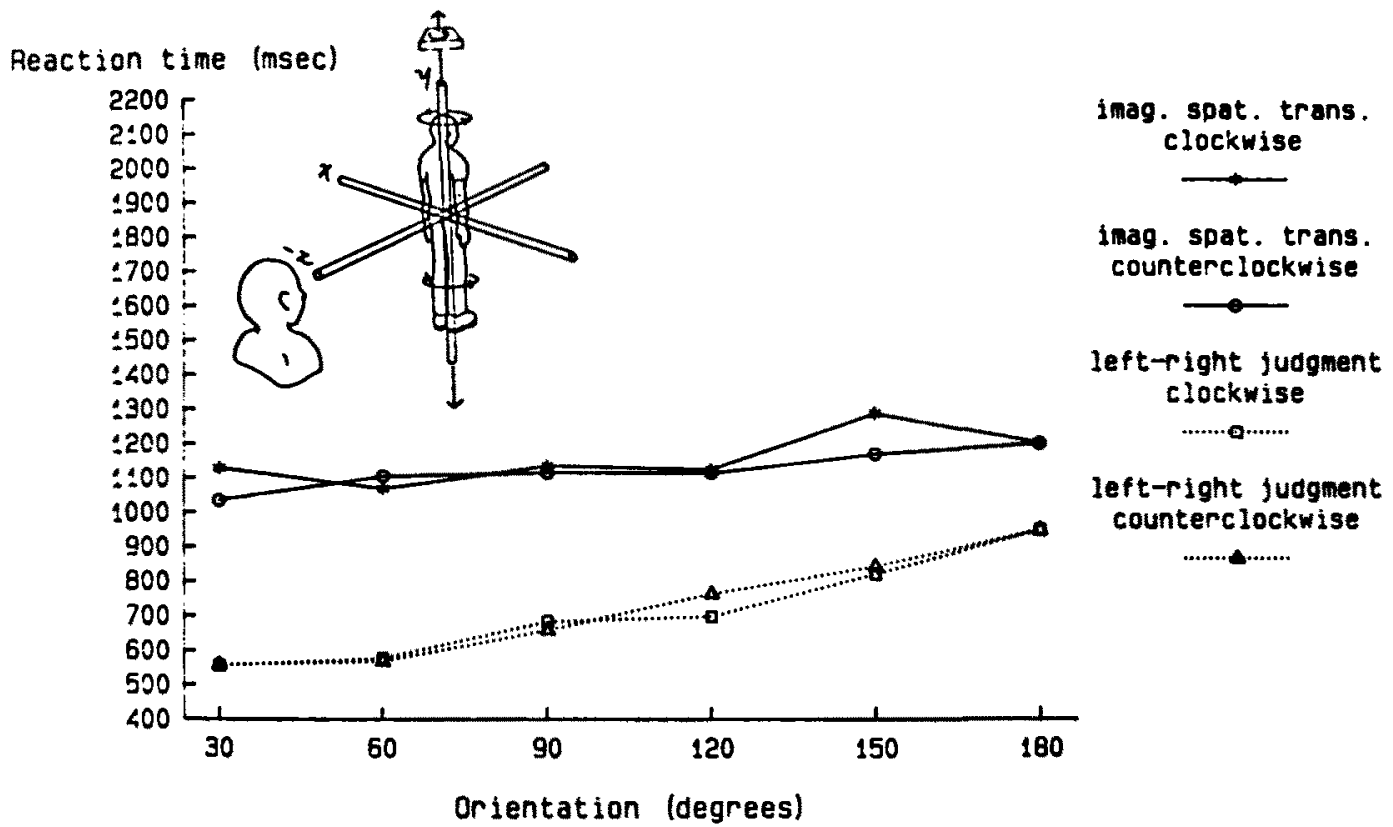

Figure 4. Left-right judgment and imagined spatial transformation for axis $(0,1,0)$ : Experiments 3 and 4. (Separate RT-OD functions for left-right judgments of the outstretched arm of the body at orientations and for imagined spatial transformations of one's body for each axis. Also shown is the location of the axes used in Experiments 3,4, and 5, and the plane or cone in which the orientation of the upper body of the stimulus lay. The origin of the coordinate system is at the center of mass [middle] of the stimulus body. Subjects' line of sight on the stimuli was always down the depicted negative $Z$ axis toward the origin [Figure 1]. For the purpose of the clearly depicting the orientation of the stimulus about an axis of rotation, the $X$ and $Z$ axes are in some cases shifted with respect to the plane of this page.)

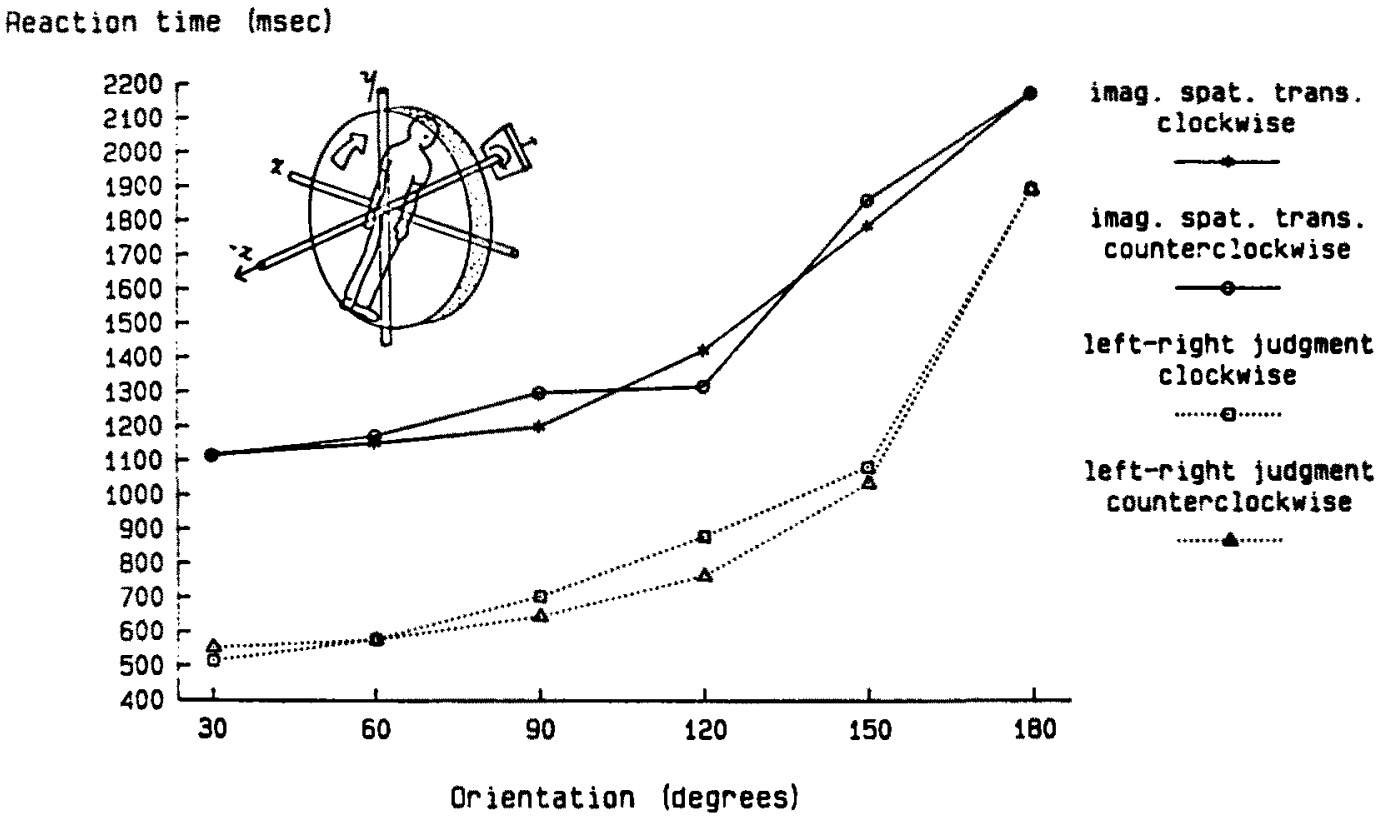

Figure 5. Left-right judgment and imagined spatial transformation for axis $(0,0,1)$ :

Experiments 3 and 4 . (See Figure 4 for explanation of format.) 


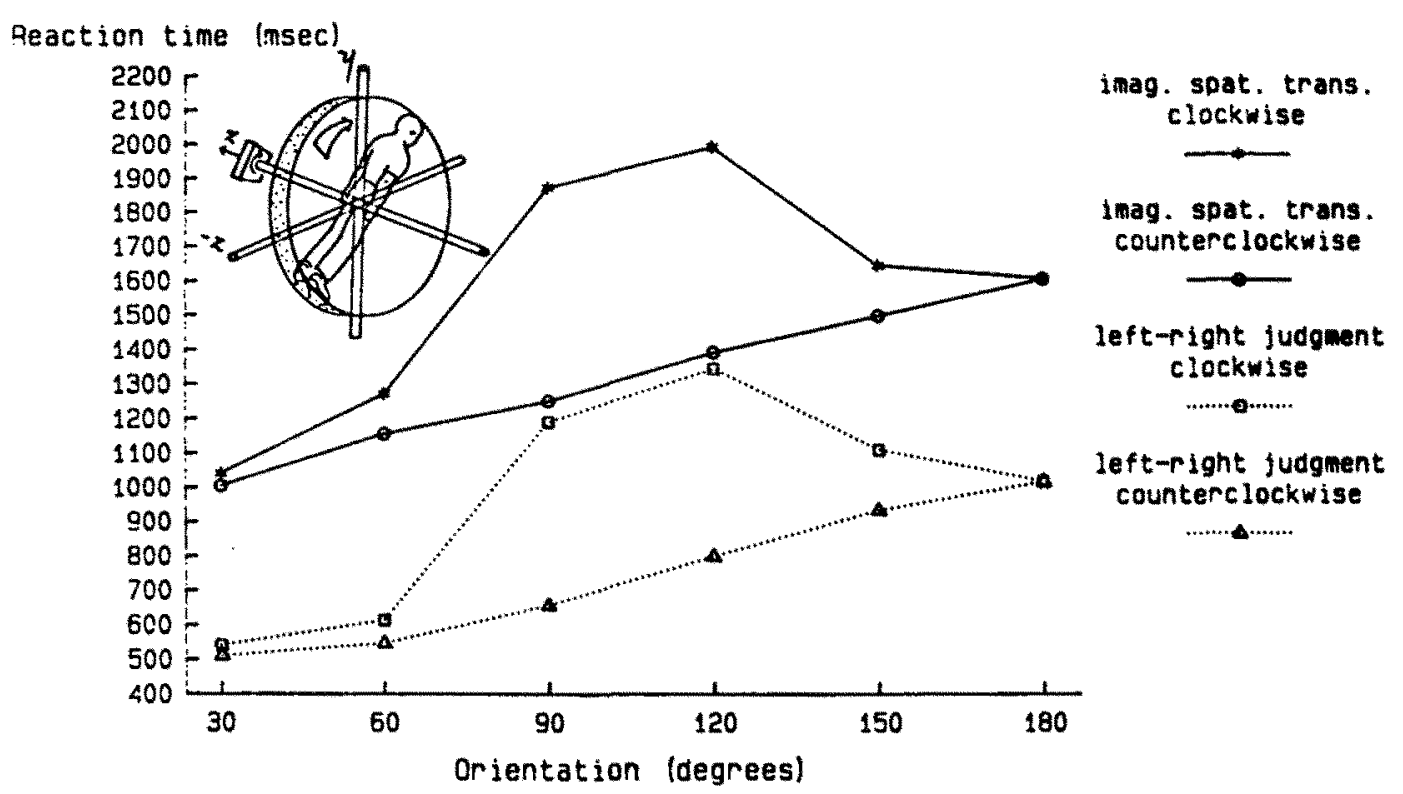

Figure 6 . Left-right judgment and imagined spatial transformation for axis $(1,0,0)$ : Experiments 3 and 4 . (See Figure 4 for explanation of format.)

slope was observed for the vertical axis (which was aligned with the body's major principal axis). The steepest slope was found for orientations in the picture plane (about the observer's line of sight). For example, RT for a $180^{\circ}$ OD varies (for different directions) from about $1 \mathrm{~s}$ (with no errors) to about $2 \mathrm{~s}$ (with an error rate of .26). RT increased markedly for stimulus orientations where the head was (a) away from observer or "forward," (b) away from observer and below the horizontal plane, (c) upside-down, and (d) upside-down, with the body nearly parallel to observer's frontal plane. However, the orientation of the head cannot explain all of the effect of stimulus orientation on RT. For example, at the upside-down orientation, for which $\mathrm{OD}$ is always $180^{\circ}$, mean RT depends on which way the stimulus body faces. Thus, for upside-down stimuli facing toward observer, RT is $1,000 \mathrm{~ms}$; facing away from observer, it is $2,000 \mathrm{~ms}$; toward observer's left, it is $1650 \mathrm{~ms}$; and towand observer's right, it is $1,500 \mathrm{~ms}$ (these means are reliably different to $p<.05$ by Newman-Keuls tests). Differences in RT for forward and backward

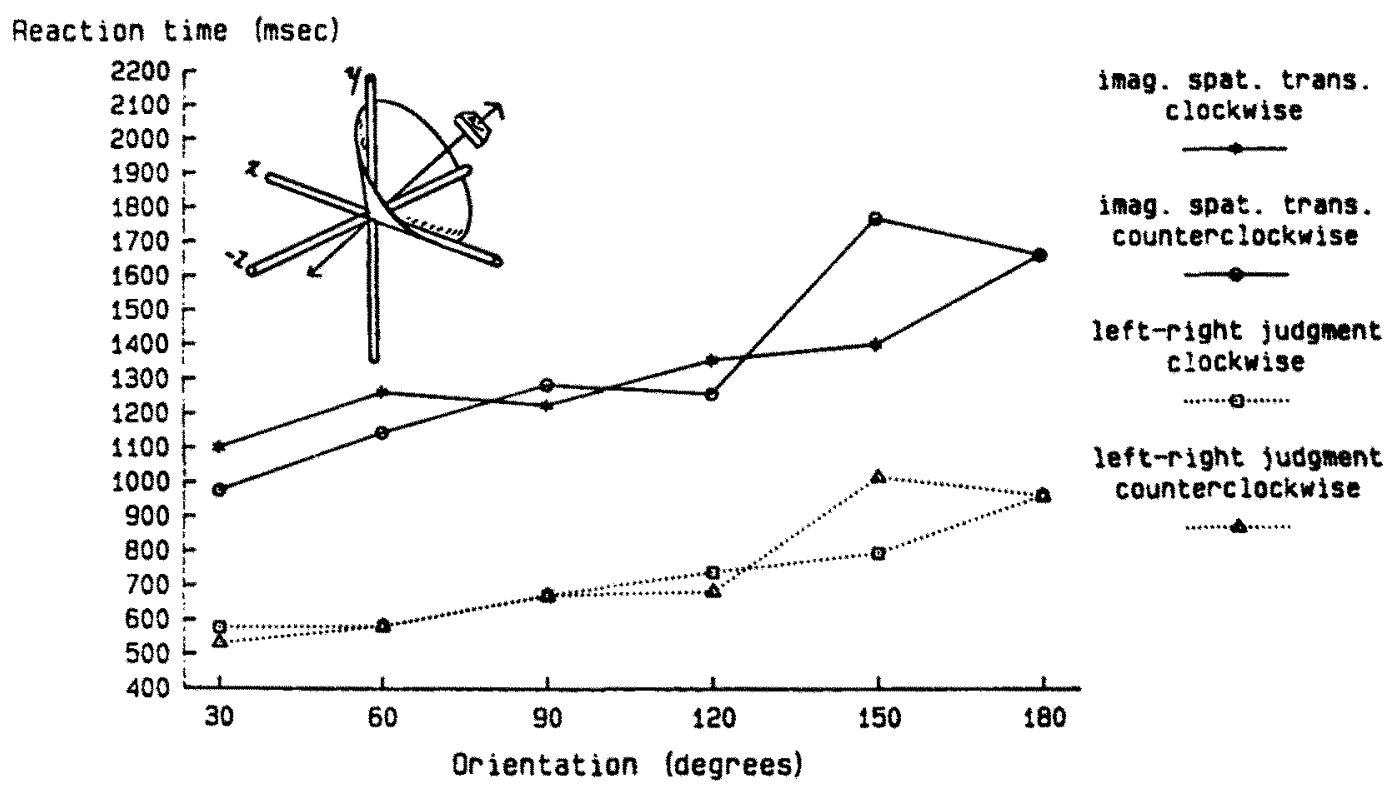

Figure 7 . Left-right judgment and imagined spatial transformation for axis $(.58, \cdots .58, .58)$ : Experiments 3 and 4 . (See Figure 4 for explanation of format.) 


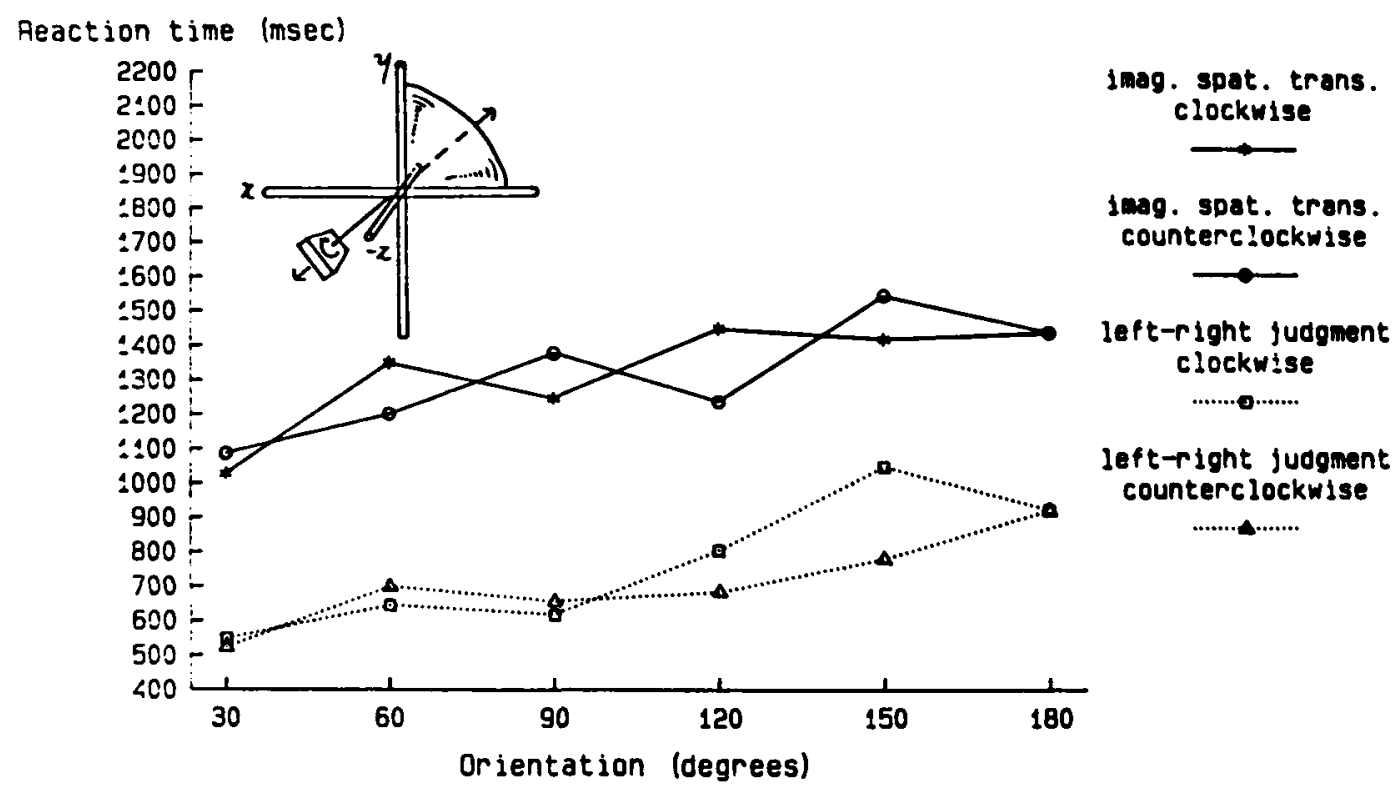

Figure 8. Left-right judgment and imagined spatial transformation for axis $(.71,-.71,0)$ : Experiments 3 and 4 . (See Figure 4 for explanation of format.)

stimulus orientations are not caused by variation in a subject's ability to detect the location of the outstretched arm. For instance, the effect is strong for stimulus orientation in the depth plane ( $[1,0,0]$ axis), where the outstretched arm is parallel to the picture plane and never occluded by the rest of the stimulus. Subjects did not move during trials, no RT patterns are not due to actual movement to stimulus orientation.

Such large differences in slopes of discriminative RT-OD functions for different planes of stimulus orientation, and for clockwise/counterclockwise directions in a plane of stimulus orientation, are unlike almost all previous findings in this area. For example, no difference was observed in slopes of discrimination functions for abstract three-dimensional shapes in picture and depth planes (Shepard \& Metzler, 1971), and only a $15 \%$ difference was observed in slopes for RT-OD functions for three planes of stimulus orientation (Metzler, 1973). However, in left-right judgments of hands and feet (Parsons, 1987a), there are comparably large (or larger) effects of direction of stimulus

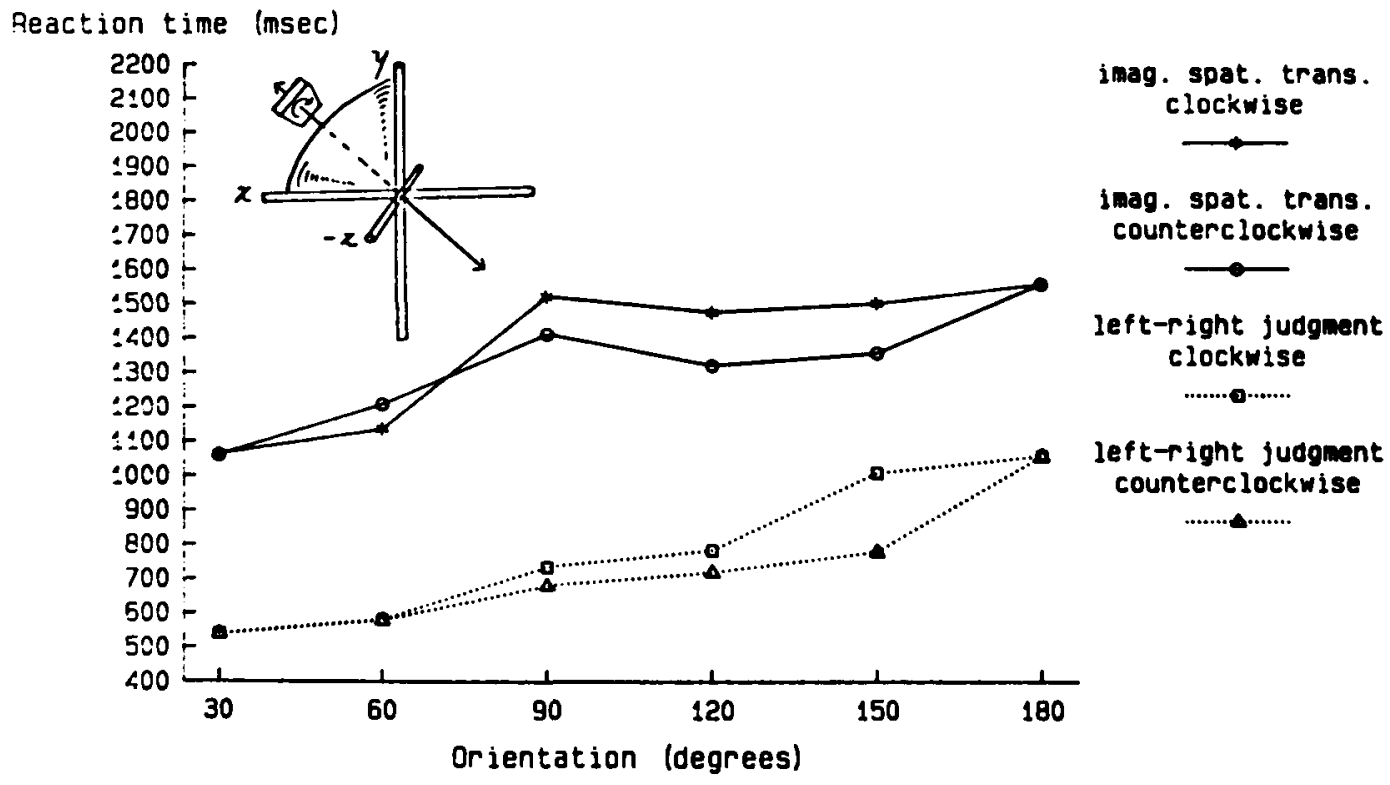

Figure 9. Left-right judgment and imagined spatial transformation for axis $(.71, .71,0)$ : Experiments 3 and 4 . (See Figure 4 for explanation of format.) 


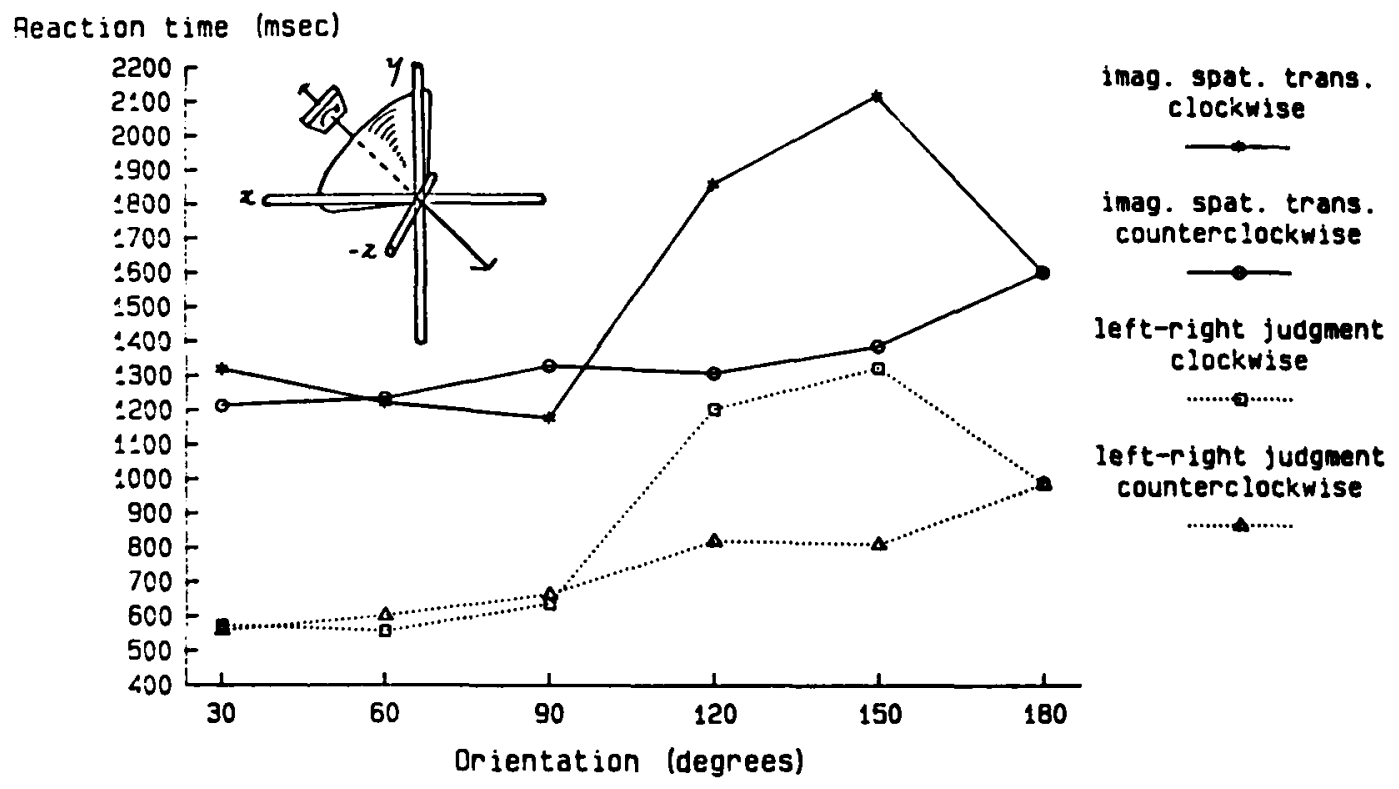

Figure 10. Lefi-right judgment and imagined spatial transformation for axis $(.58, .58, .58)$ : Experiments 3 and 4. (See Figure 4 for explanation of format.)

orientation. In addition, in a study of the discrimination of abstract three-dimensional objects, the slope of resulting discrimination functions varied by a factor of more than 2 across different planes of OD (Parsons, in press).

\section{Experiments 4 and 5: Imagined Spatial Transformations of One's Body}

Experiments 4 and 5 collected evidence on the following hypothesis about subjects' left-right judgments in Experiments 1 , 2 , and 3 .
1. The effect of stimulus orientation on RT is due mostly to its effect on properties of imagined spatial transformations rather than on comparison of shape involved in the discrimination.

2. Subjects imagine a representation of their upright body rotating into the orientation of stimuli.

3. The spatial origin of these imagined spatial transformations is the subject's own upright orientation during Experiments 1,2 , and 3 .

These experiments use a paradigm in which each subject is

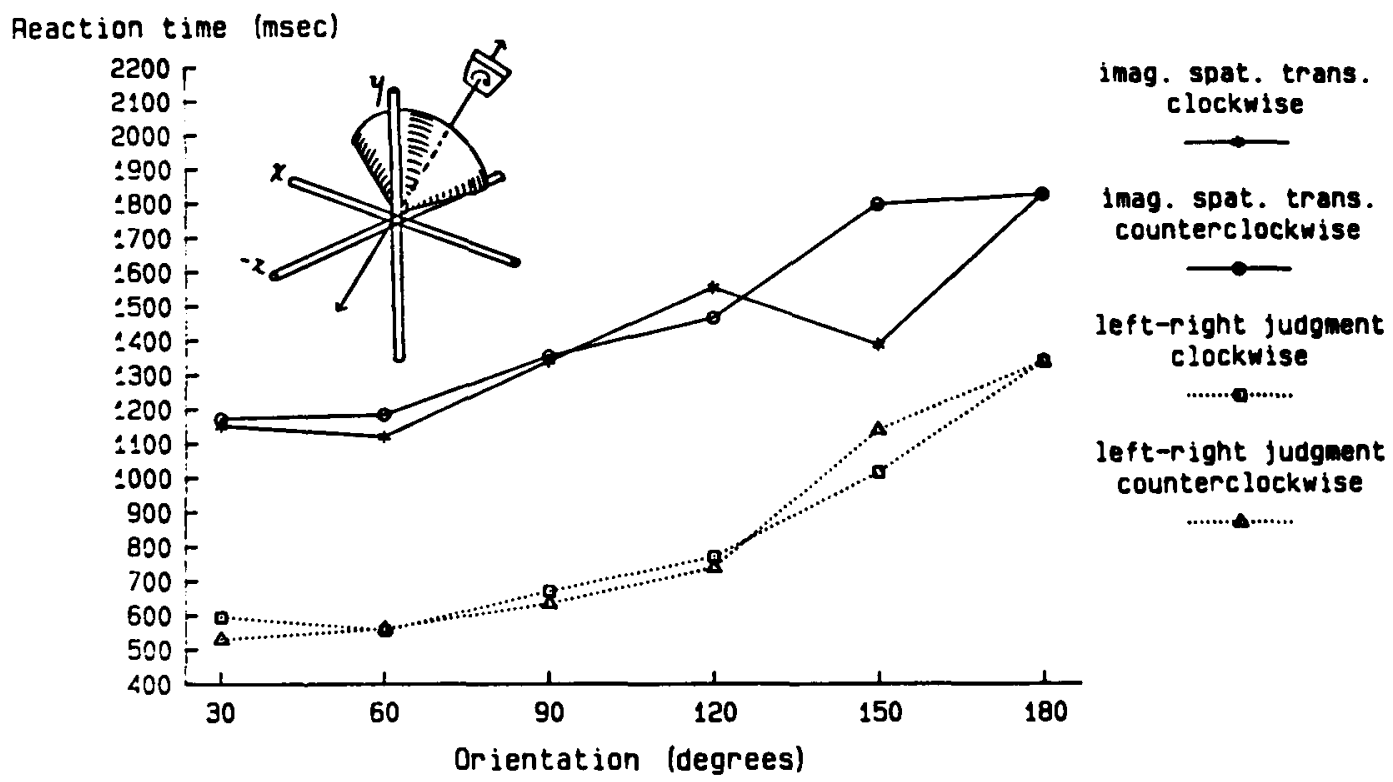

Figure 11. Left-right judgment and imagined spatial transformation for axis $(0, .71, .71)$ : Experiments 3 and 4. (See Figure 4 for explanation of format.) 


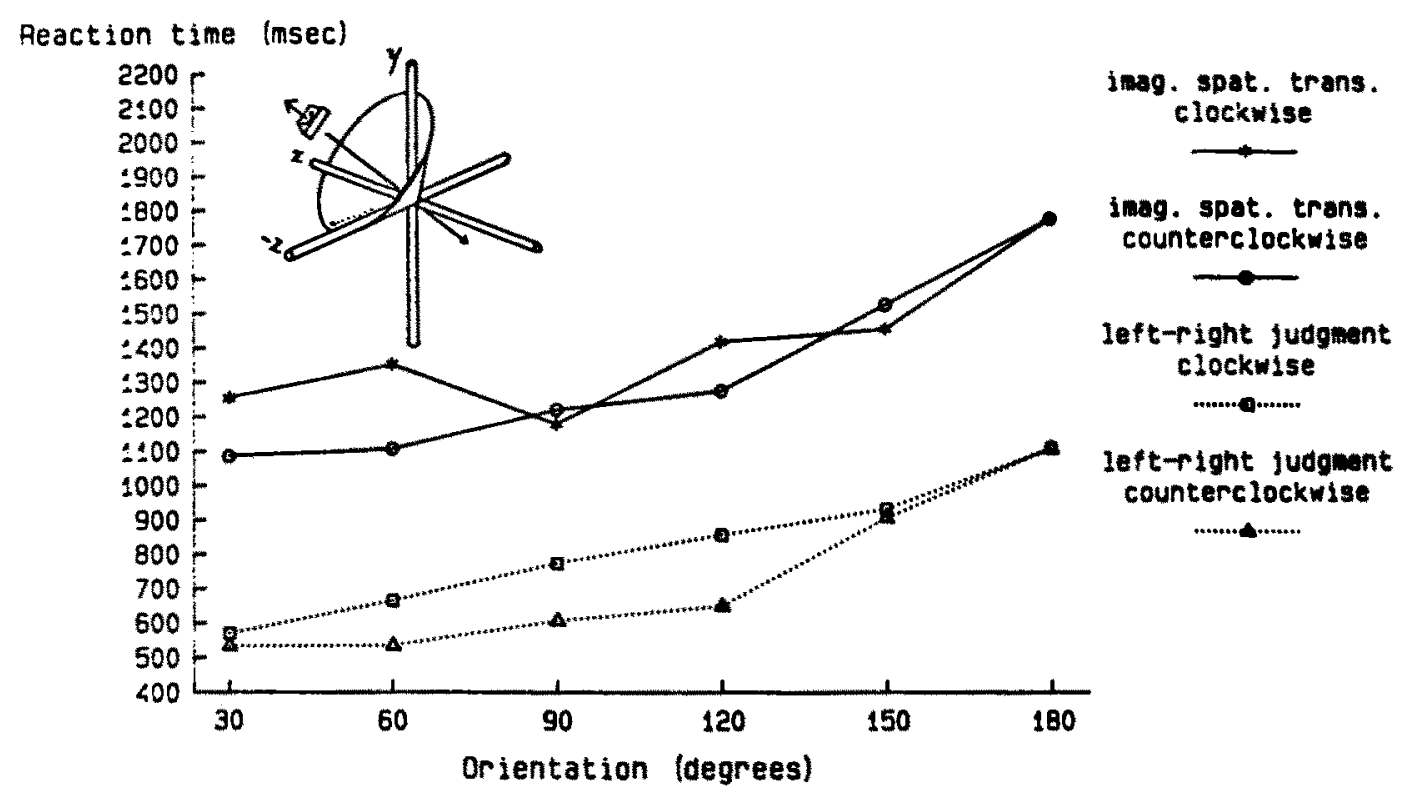

Figure 12. Left-right judgment and imagined spatial transformation for axis $(.58, .58,-.58)$ : Experimen is 3 and 4. (See Figure 4 for explanation of format.)

instructed to imagine his or her body and arm rotating from their orientations during the task to the orientation of a stimulus, and to say "Now" when the imagined spatial transformation is complete. Trials are blocked so that stimulus handedness is known in advance of each trial, and no left-right judgment of a stimulus is required. In Experiment 4, this paradigm is used with the stimuli from Experiment 3, and subjects are free to imagine any path between their own orientations and that of the stimulus. In Experiment 5, subjects are instructed to imagine themselves rotating between their own orientations and that of the stimulus using experimenter-specified shortest paths. Thus, unlike in Experiments 1-4, in Experiment 5, the extent of path is restricted; this enables assessment of the rate of imagined spatial transformations in different planes.

These two simulation paradigms are reminiscent of those in which people imagine an object's rotation in a specific plane in response to a cue providing preparatory information about an upcoming trial (e.g., Cooper, 1975; Cooper Shepard, 1975;

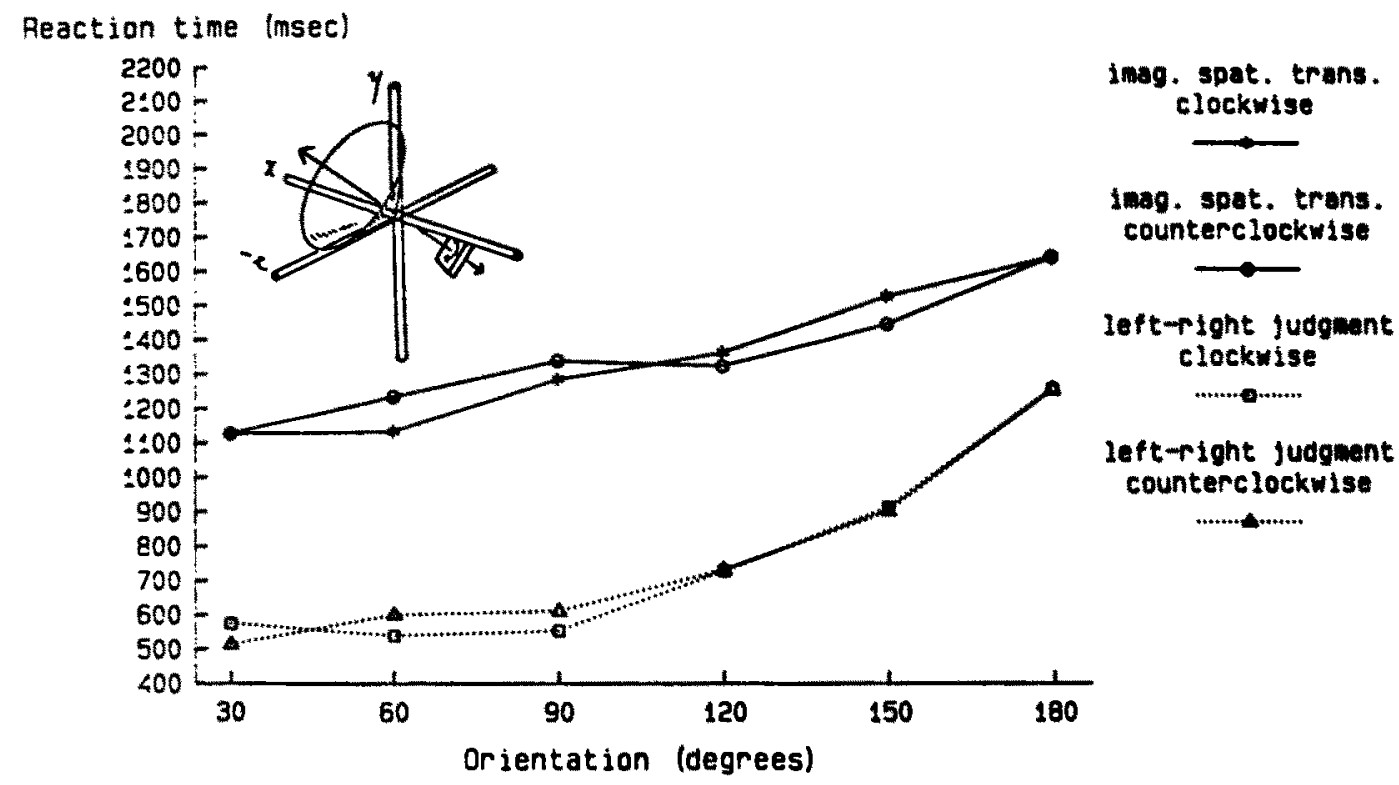

Figure 13. Left-right judgment and imagined spatial transformation for axis $(0,-71,71)$ : Experiments 3 and 4 . (See Figure 4 for explanation of format) 


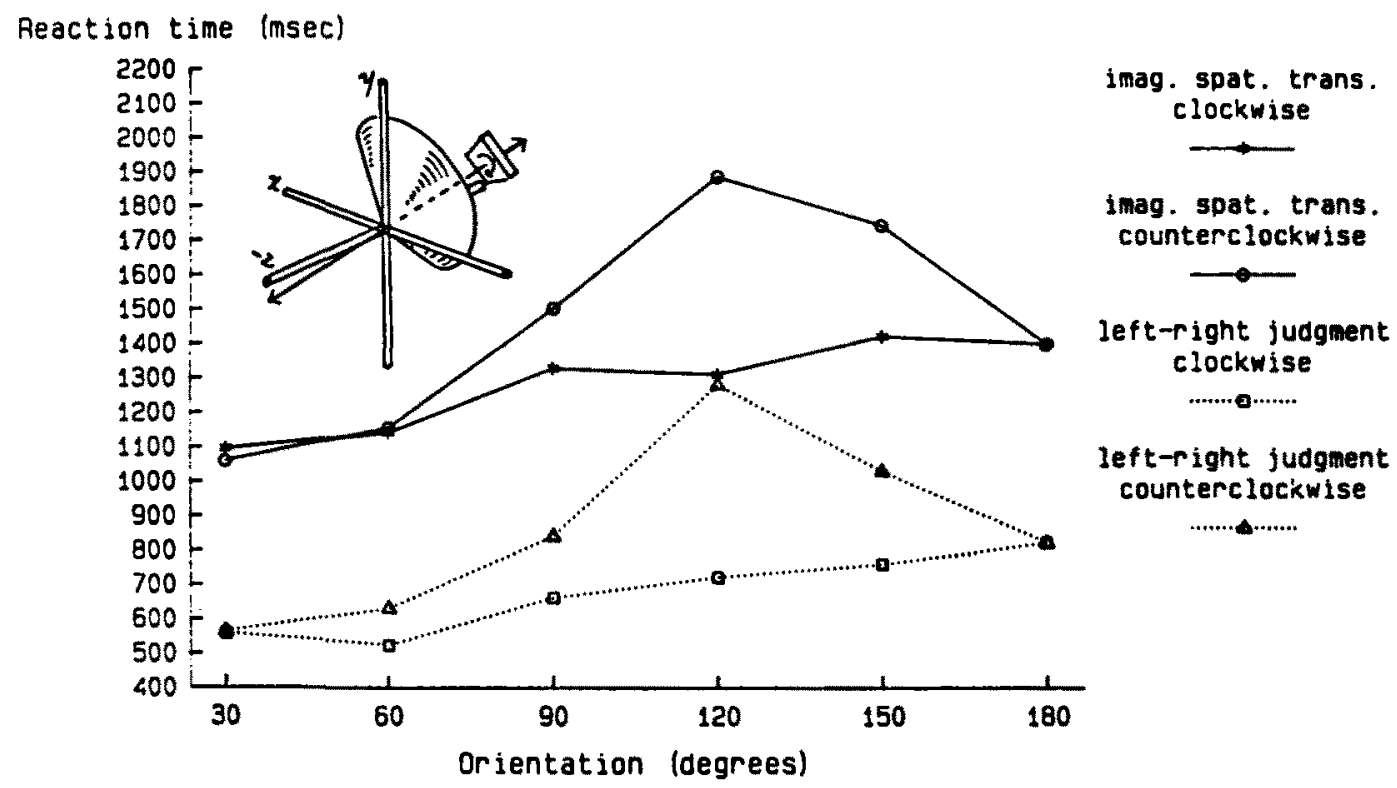

Figure 14. Left-right judgment and imagined spatial transformation for axis $(-.58, .58, .58)$ : Experiments 3 and 4 . (See Figure 4 for explanation of format.)

Metzler, 1973). The preparation-time/orientation function that is observed has a linearly increasing slope very similar to that for the RT-OD function for discriminating mirror-image objects (Cooper, 1975). In a study like Experiment 4, RT-OD functions for left-right judgments of a hand or foot were strongly correlated with the time to imagine spatial transformation of one's hand or foot from its orientation during the task to the orientation of stimuli (Parsons, 1987a). However, differences in RT patterns for various planes of orientation difference in Par- sons' study were slightly less extreme for simulation than for the discrimination paradigm. Also, the overall mean RT was longer for the simulation than discrimination task.

Of interest in Experiment 4 is whether a subject imagining his or her body (and arm) moving between pairs of orientation differences like those in Experiment 3, produce comparable RT-OD functions. If so, this result is good evidence for the foregoing three hypotheses. A strength of this paradigm is that the relation between sets of RT-OD functions in Experiments 3 and

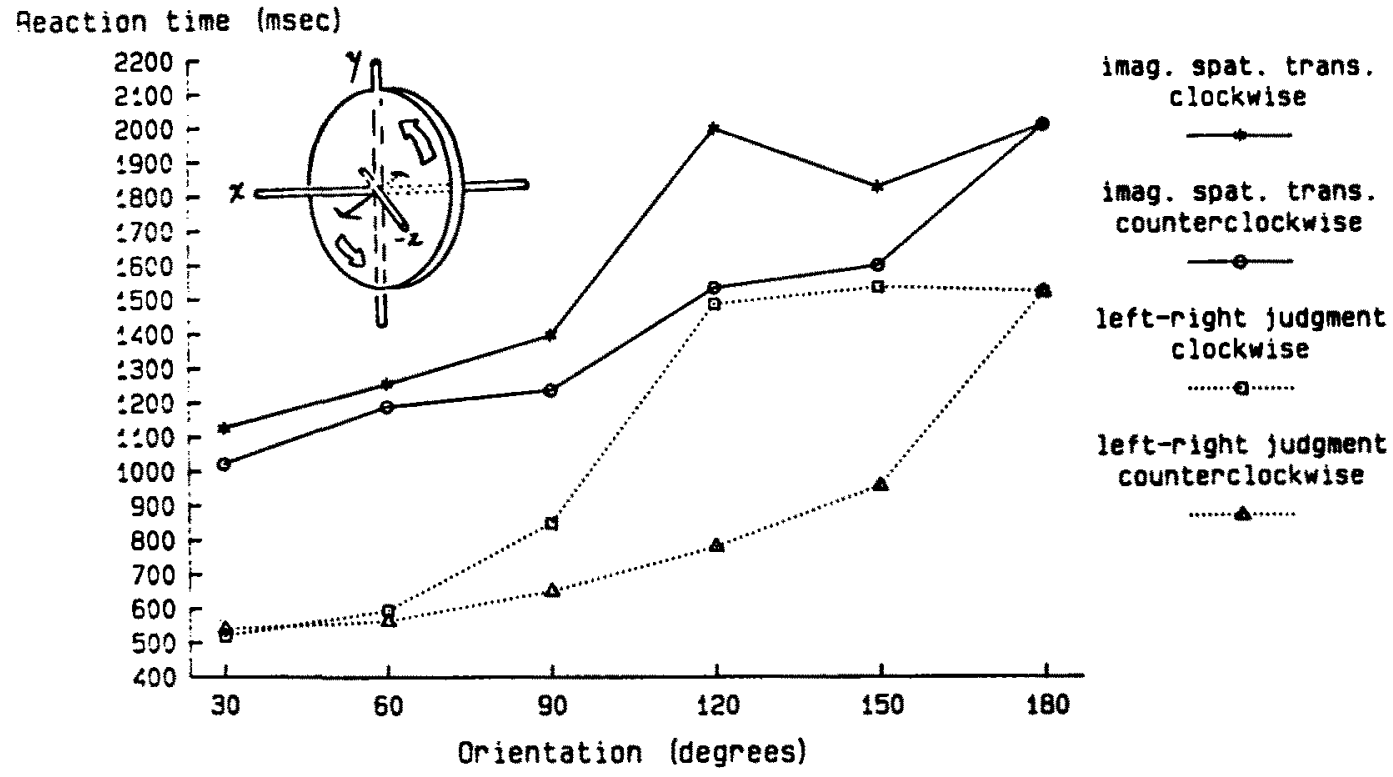

Figure 15. Left-right judgment and imagined spatial transformation for axis $(.71,0,-.71)$ : Experiments 3 and 4. (See Figure 4 for explanation of format.) 


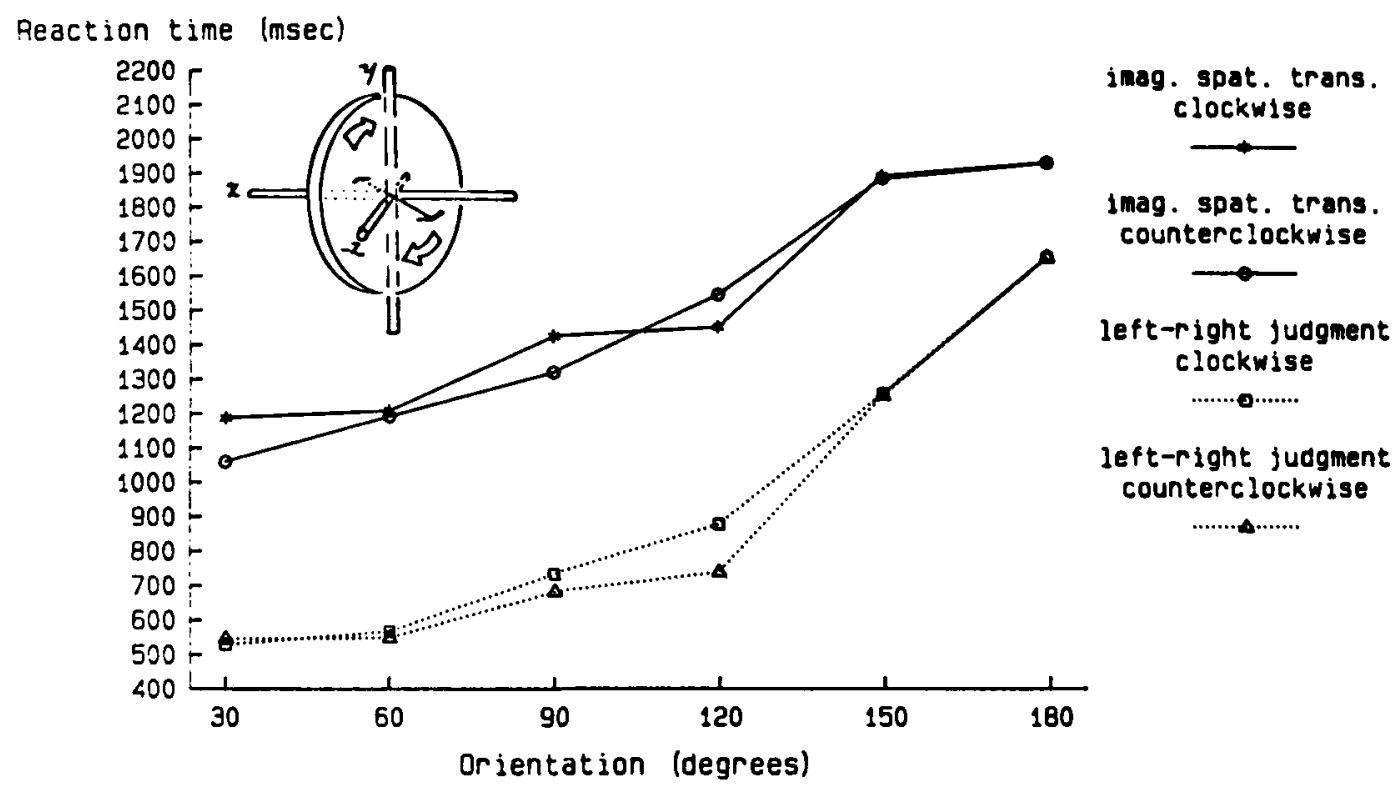

Figure I6. Left-right judgment and imagined spatial transformation for axis $(.71,0, .71)$ : Experiments 3 and 4. (See Figure 4 for explanation of format.)

4 could in principle reveal whether the origin of imagined spatial transformation in Experiment 3 is different by as much as $30^{\circ}$ in any direction from the origin subjects are instructed to use (cf. Parsons, 1987a). However, in light of the variability of RTs in this paradigm, Experiment 4 has the power to detect a minimum disparity of $60^{\circ}$ between the task orientation subjects are instructed to use as a spatial origin here and the spatial origin of the subjects' spatial transformation in Experiment 3.

\section{Experiment 4: Imagined Spatial Transformation of One's Body via the Path of One's Choice}

\section{Method}

Subjecls. A total of 13 right-handed MIT undergraduates who had not been in any related experiments participated for $\$ 5$ an hour.

Stimuli. design, and procedure. All aspects of stimuli, design, and procedure are identical to Experiment 3 except the following. Trials were grouped by the handedness of the stimuli: 36 trials with left-hand stimuli were alternated with 36 trials with right-hand stimuli. Within each group of 36 trials, the axis and angle of rotation were randomly ordered with an approximately equal number of trials for each angle and axis. After 20 randomly selected practice trials, a single replication of all 288 trials was performed. (Subjects were not instructed as to when they should imagine stretching out their arm.) They knew the stimulus handedness before each trial. Their vocal responses triggered a voiceactivated relay.

\section{Results}

Subjects reported having no difficulty following instructions and imagining their bodies and arms passing from their orientation during the task to the orientation of stimulus. Time to imagine the spatial transformation depended on how much the stimulus orientation differed from that of the subject. However, it also depended on which axis was used to create the orienta- tion difference between the stimulus and subject. An ANOVA of RTs was conducted with 11 ODs, stimulus handedness, and axis. RT depended on axis, $F(12,144)=10.45, p<.0001$, and OD, $F(10,120)=29.27, p<.0001$. The effect of OD on RT depended on axis, $F(120,1440)=3.60, p<.0001$; RT for different axes depended on stimulus handedness, $F(12,144)=$ $1.89, p<.05$; and the effect of OD on RT depended on both axis and stimulus handedness, $F(120,1440)=1.68, p<.0001$.

Slopes of the best fit linear regressions of RT on OD for individual planes of orientation varied by a factor of 5 . For the most part, this striking range in slopes reflects the tendency for imagined spatial transformation times to be longer for orientations near the upside-down orientation, independent of $O D$. As shown in Figures 4-16, slopes and shapes of functions for different planes of orientation are very similar to those in Experiment 3. Furthermore, as in Experiment 3, RT-OD functions for several axes are different for clockwise and counterclockwise ODs, which reflects the tendency for longer RTs to orientations where the head pointed forward (i.e., away from observer) and forward or below horizontal plane.

As in Experiments 1 and 3, the evidence is less consistent with a rotations-by-dimensions procedure than with spin-precession and shortest-path procedures. However, because of the strong effect on RT of the direction of $O D$, none of these simple models are very predictive. On the basis of linear regression of RT means on $\mathrm{OD}, 40 \%, 52 \%$, and $54 \%$ of the variance is accounted for by assuming a rotations-by-dimensions, spin-precession, or shortest-path procedure, respectively.

Comparison to RTS in Experiment 3. The ANOVA results are like those for left-right judgments of the same stimuli in Experiment 3. The two sets of RTs are correlated at $.92, F(1$, $142)=732.98, p<.0001$. The differences for RTs to Experiments 3 and 4 are confined to the overall mean (intercept) of RTs, which is about $500 \mathrm{~ms}$ longer here than in Experiment 3. 
A comparable difference in RT was found in corresponding discrimination and simulation experiments by using left and right hands and feet (Parsons, 1987a). At least some of this difference is likely due to the fact that subjects in Experiment 3 had three times as much practice at the task. When subjects are as practiced at a discrimination task as at the corresponding preparation or simulation task, overall means for the two tasks are approximately equal (e.g., Cooper, 1975).

These similar RT patterns in Experiments 3 and 4 suggest that the discrimination and simulation tasks require similar processes for responding to the orientation differences of stimuli. The differences in RT across the two experiments appear to be unrelated to the orientation difference between stimuli and subjects. It is possible that the simulation task induces greater time for some kind of information processing before and/or after an imagined spatial transformation. Further research is necessary to identify in detail the nature of the mental processes used in these two tasks.

\section{Discussion}

Time for an imagined spatial transformation depends on its direction. These results show that the time to imagine spatial transformations of an object (i.e., a representation of one's body) can depend very strongly on the orientation to which, or through which, it passes. Most previous work on imagination of other objects found effects only of the extent of OD (e.g., Cooper, 1975; Cooper \& Shepard, 1975; Metzler, 1973). These findings are consistent with those of imagined spatial transformations of one's hands and feet (Parsons, 1987a).

Model of performance. The high correlation between time for imagined spatial transformations and for left-right judgments $(\mathrm{r}=.92)$, confirms the performance model used to interpret the results of Experiments 1, 2, and 3. Furthermore, RTOD functions observed here are less consistent with the use of an inefficient procedure like rotations by dimensions than with the use of efficient procedures like spin precession or shortest path. Use of a rotations-by-dimensions procedure would require assuming that rates and initiation times vary (with direction of $O D$ ) by considerably greater amounts than for the other two procedures. This is confirmed by the goodness of fit of linear regressions.

Overall, these results fit several different explanations. Stimulus orientation may affect the time for an imagined spatial transformation by influencing one or more of either its rate, path, or initiation time.

1. Suppose (a) people use very efficient paths here (so there is only a small amount of variation in extent of imagined path for ODs in different directions). Also suppose (b) initiation times of imagined spatial transformations are constant for all ODs. If so, then these results would suggest that rates of imagined spatial transformations can vary much more than results of other studies have indicated.

2. Alternatively, suppose extent of the imagined path for an OD varies in different directions, but rates or initiation times are constant. The results then suggest that people use quite different paths for the same extent of OD. This conclusion is consistent with observations of imagined spatial transforma- tions of one's hands and feet, which suggest that paths of different extent are imagined for different directions of $O D$.

3. If rates and extent of path do not vary for the same $O D$ in different directions, then results suggest that initiation time (i.e., time to plan or find an efficient path) would vary greatly.

Other possibilities involve some combination of the preceding possibilities. In addition, it is also possible that subjects use a mixture of strategies for stimuli at different orientation differences. This could entail variation in the extent, rate, and initiation time of imagined spatial transformations. Paradigms with more precise control and measures of performance, like that in Experiment 5, are required to sort out these various possibilities.

Time for imagined spatial transformation depends slightly on which arm is outstretched. Effects involving stimulus or response handedness were found in Experiment 3 where the leftright judgment was indicated with a left- or right-hand buttonpress response. Because such effects were also observed in Experiment 4 , where no left-right judgment (or response) was made, these handedness effects must be related to stimulus handedness rather than to response handedness. Handedness effects are observed for some of the $90^{\circ}$ or greater ODs, in clockwise and counterclockwise directions, for 9 of 13 axes. In some cases, RT for the left-hand stimulus is longer than for the righthand stimulus, and in other cases the reverse is true. However, there are no simple or uniform patterns for different planes of orientation. The rate, the path, or the initiation time of imagined spatial transformation of the body is influenced by which arm is outstretched.

\section{Experiment 5: Imagined Spatial Transformations of One's Body via Shortest Path}

This experiment examines the rate of imagined spatial transformations by using a simulation paradigm like that in Experiment 4. Its purpose is to evaluate how rate is influenced by the orientation to which subjects imagine themselves passing. Subjects are instructed to imagine their body passing in shortest paths from their orientation during the task to the stimulus orientation. They are told, for example, that for the next 11 trials the stimulus will show the left arm outstretched and that the body will be upright but at any orientation about the vertical axis.

The set of planes or axes used to create ODs in this experiment was selected so that it was representative of the possible orientations of the body, and the associated paths and orientations could be readily demonstrated, so as to facilitate subjects' ability to accurately imagine the spatial transformations. Despite the fact that the set of orientations about the $(-.58, .58$, .58 ) axis cannot be simply described (Figure 14 ), such orientations were used because there were considerable RT differences for clockwise and counterclockwise ODs about this axis. In addition, this latter axis was used because the extent of path for the rotations-by-dimensions procedure is greater than for the spin-precession and shortest-path procedures (the extents of which are fairly similar: cf. Table A-2 in Appendix).

Thus, if subjects follow instructions, they will be imagining spatial transformations of the same extent for the corresponding extent of orientation difference. Furthermore, there should 
be little variation in initiation times for imagined spatial transformations in different directions: Initiation time should be little influenced by differential uncertainty in, or familiarity with, location of path. (However, in at least some cases, this assumption may turn out to be unwarranted if subjects have difficulty learning those paths that are difficult to describe.) If these assumptions are correct and no other factors are involved, then shape or slope of functions for different planes of orientation directly reflect the rate of imagined spatial transformations.

If subjects produce RT patterns very similar to those in Experiments 3 and 4, it would suggest that subjects in Experiments 3 and 4 imagined very efficient paths. There is little or no difference in RT functions predicted by spin-precession and shortest-path procedures here, so RTs cannot determine which procedure was used in Experiments 3 and 4. In some cases, intercept differences are predicted for the use of the rotations-bydimension procedure as compared with the use of spin-precession and shortest-path procedures (Table A-2). These differences may be verified by statistical test.

\section{Method}

Subjects. A total of 14 right-handed MIT undergraduates, who had not been in any related experiments, participated for $\$ 5$ an hour.

Stimuli and design. Stimuli were exactly like those in Experiment 4, except that only those produced by $(1,0,0),(0,1,0),(0,0,1),(.71,0$, $.71),(.71,0,-.71)$, and $(-.58, .58, .58)$ axes were used. The 132 practice trials were designed exactly like those in Experiment 4. The 132 test trials (in random, unique order for each subject) were organized so that 33 trials with the left arm of stimulus outstretched were followed by 33 trials with the right arm outstretched. These 33 trials were composed of three sets of 11 trials, each set with a different plane of $O D$. This was followed by a comparable set of trials with the right arm outstretched. Finally, two more sets of 33 trials were performed in the same manner. Each possible kind of trial was performed only once.

Procedure. On the practice trials, the procedure was exactly like that in Experiment 4. Before each test trial, subjects knew which arm was outstretched and what plane (or axis) they were to use. They had to determine in which direction to imagine a rotation after they saw each stimulus (e.g., clockwise or counterclockwise about the axis of rotation). Before each set of 11 trials, the experimenter demonstrated the stimulus orientations with a human-like doll. Subjects were then asked to imagine themselves (with appropriate arm outstretched) at a few orientations of their own choice (about that axis). Then, they performed 11 trials. The procedure was otherwise identical to Experiment 4.

\section{Results}

Subjects reported having difficulty learning the paths for orientations created by rotations about the $(-.58, .58, .58)$ axis, and, to a lesser extent, the $(.7,0, .7)$ and $(.7,0,-.7)$ axes. Otherwise, they reported following instructions. Overall RT increased with the difference between the orientation of the subject and of the stimulus. It aiso depended on the direction of the OD. An ANOVA of RTs that used axis, 12 ODs, and stimulus handedness showed that RT depended on axis, $F(5,65)=8.99$, $p<.001$, and $\mathrm{OD}, F(10,130)=6.49, p<.001$.

Slopes of the best fit linear regressions of RT on OD for different planes of orientation varied considerably (by a factor of 2.5). Also, RT-OD functions were different for clockwise and counterclockwise ODs about $(1,0,0),(.71,0, .71)$, and $(-.58$, $.58, .58)$ axes.

Comparison with RTs in Experiments 3 and 4. The correlation of RT at ODs created by rotation about axes $(1,0,0),(0$, $1,0),(0,0,1),(.71,0, .71)$, and $(.71,0,-.71)$ with those in Experiment 3 are $.90, .96, .95, .86, .88$ (for each, $p<.001$, $d f=$ 9), respectively. With RTs in Experiment 4, these correlations are $.88, .71, .97, .93, .89(p<.001$, except $r=.71$ for which $p<$ $.02)$. For ODs created by rotations about $(-.58, .58, .58)$ axis, this correlation for Experiment 3 is .73 and for Experiment 4 is .77 ; without $\mathrm{RT}$ at $120^{\circ}$ clockwise $\mathrm{OD}$, these correlations are .96 and $.90(p<.001)$.

The overall correlation of these RTs with (a) those in Experiment 3 is $.60, F(1,64)=35.13, p<.0001$, and with (b) those in Experiment 4 is $.68, F(1,64)=54.56, p<.0001$. This relatively weak correlation occurs because the relation of intercepts (or overall mean RTs) among the individual members of the set of axes in Experiment 3 is different from those among their counterparts in Experiments 3 and 4. Mean RT for $(.71,0, .71)$, $(.71,0,-.71)$, and $(-.58, .58, .58)$ axes here are relatively longer than in Experiment 3. Compared with Experiment 4, means for $(0,0,1)$ and $(1,0,0)$ axes here are relatively shorter, and those for $(.71,0, .71)$ and $(.71,0,-.71)$ axes are relatively longer.

Overall mean RT is comparable to that in Experiment 4 and is about $500 \mathrm{~ms}$ longer than in Experiment 3.

\section{Discussion}

Rate of imagined spatial transformation varies in different directions. On the assumption that no other factors influenced the slope of the RT-OD functions, these results demonstrate that the rate of imagined spatial transformations depends on the orientations to or through which one's body is imagined to pass. The rates of imagined transformations range from $300^{\circ}-700^{\circ}$ per second. These rates fit as expected in the range of rates observed for stimuli of varying complexity or familiarity. For abstract, three-dimensional objects (like those used by Shepard \& Metzler, 1971), the inferred rates vary from $50^{\circ}$ to $100^{\circ}$ per second; for experimentally familiar random polygons, $400^{\circ}-600^{\circ}$ per second (Cooper, 1975); for letters and numbers, $600^{\circ}$ per second (Cooper \& Shepard, 1973; Hinton \& Parsons, 1981); and for one's own hand and foot, apparently from $300^{\circ}$ to $900^{\circ}$ per second (Parsons, 1987a).

The results reported here do not indicate exactly why the rate of imagined spatial transformations varies for different directions. Two possibilities are subjects' familiarity with orientations of their body and how well practiced a spatial transformation to an orientation is. If upside-down orientations are less familiar than upright orientations, then it is possible that, for unfamiliar orientations, people imagine less efficient paths, take longer to find a path, or produce slower imagined spatial transformations. For example, the fast rate for the vertical axis (which was aligned with the axis of elongation of the body) may be accounted for by two hypotheses. First, rotation about the vertical is probably the most common of all rotations in our ecology, and familiarity may increase the rate of imagined rotation. There is some evidence that the effect of practice on rate of imagined rotations may be profound. Kaushall \& Parsons (1981) found that with practice the slope of the RT-OD function 
for discriminating identical and mirror-image pairs of differently oriented, abstract, three-dimensional objects gradually declined to zero (though most subjects reported imagining the reorientation of the stimuli throughout the experiment.) Second, rotation about the major principal axis of an object (i.e., the axis of elongation of the body) may be more rapid than rotation about other axes (Metzler, 1973).

Another possibility is that imagined spatial transformation of one's body simulates, to some extent, dynamic properties of actual motion of the body; if so, imagined spatial transformations to orientations near upside-down could be expected to be longer than to orientations near upright. An analogous account is suggested by studies of imagined spatial transformations of one's hands and feet (Parsons, 1983b, 1987a). There may be other factors involved, but they remain to be discovered.

Effect of intermediate versus final orientation. There is some evidence here that the rate of an imagined spatial transformation is influenced more by its final orientation than by its intermediate orientations. Consider, for example, the functions for orientations in the depth plane ([1, 0, 0] axis): RT peaks at the $120^{\circ}$ clockwise $\mathrm{OD}$, and declines to $180^{\circ} \mathrm{OD}$. If subjects imagine their bodies passing in shortest paths, then when they imagine their bodies at $150^{\circ} \mathrm{OD}$, they must have (briefly) imagined themselves at $120^{\circ} \mathrm{OD}$ orientation. This assumes that imagined spatial transformations represent approximately continuous intermediate orientations of an object, as the findings of Cooper (1976) and Metzler (1973) indicated. However, the time to imagine one's self at the $120^{\circ} \mathrm{OD}$ is longer than that at the $150^{\circ}$ $O D$, even though the latter is of greater extent and presumably passes through the former orientation. (It is possible, although unlikely, that subjects, contrary to instruction, did not imagine a shortest path in such a case. Instead, they could have imagined rotation counterclockwise (backward), the long way about the axis, passing through $180^{\circ} \mathrm{OD}$ to $150^{\circ} \mathrm{OD}$.) A related but converse effect may be seen in the RT-OD function for imagined spatial transformations in the frontal plane $([0,0,1]$ axis, Figure 4$)$. The rate of imagined spatial transformation is much less for $30^{\circ}-150^{\circ}$ ODs than for the $180^{\circ} \mathrm{OD}$. Therefore, rate could be uniformly slow to $180^{\circ} \mathrm{ODs}$ (when it is the final orientation), and uniformly fast to $150^{\circ} \mathrm{ODs}$ (when it is the final orientation). This could be consistent with the interpretation discussed earlier of different rates for orientations in clockwise and counterclockwise directions. Alternatively, the rate of imagined spatial transformations to the $150^{\circ} \mathrm{OD}$ could (always) be relatively fast, and the rate from the orientation at the $150^{\circ} \mathrm{OD}$ to that at the $180^{\circ}$ OD could (always) be slow.

Geometrical basis of imagined spatial transformations. For the most part, RT-OD functions produced by subjects given explicit training and instructions for shortest paths are very similar to those for left-right judgments for the same stimuli (and observer orientation). Furthermore, the functions in this last experiment are similar to those for imagined spatial transformations (with the same observer and stimulus orientation) for which subjects are free to imagine any path. This similarity of functions in Experiments 3, 4, and 5 suggests that subjects spontaneously used very efficient (e.g., spin-precession or shortest) paths for those orientations in Experiments 3 and 4. The results are not consistent with the hypothesis that subjects in Experi- ments 3 and 4 used an inefficient spatial transformation procedure such as rotations by dimensions.

Subjects' difficulties learning shortest paths. The two main differences between RT-OD functions here and in Experiments 3 and 4 may have resulted from unwanted side effects of the instruction set and because paths to some of the stimulus orientations were difficult to memorize. First, the function in Experiment 5 for the $(-.58, .58, .58)$ axis is different from those in Experiments 3 and 4 only in that RT at $120^{\circ}$ clockwise OD is relatively short (by about $550 \mathrm{~ms}$ ). Unfortunately, this short RT may be an effect of instructions familiarizing subjects with the body at this orientation. The $120^{\circ} \mathrm{OD}$ is the only orientation about this axis at which the body is in a principal plane of observer's visual environment. This orientation was used to illustrate the plane (or cone) of orientations relative to observer's orientation that are difficult to describe (Figure 14). There is also a difference in relations in the overall $\mathrm{RT}$ for each axis: Overall mean RT for the $(.7,0, .7)$ and $(.7,0,-.7)$ axes are longer here than in Experiments 3 and 4. This may have resulted if slower initiation times were caused by subjects' difficulty in using a certain plane of orientation. Subjects also found orientations for the $(-.58, .58, .58)$ axis difficult to memorize. If RT for the $120^{\circ}$ clockwise $O D$ about $(-.58, .58, .58)$ axis had been comparable to that in Experiments 3 and 4 (as RTs for each of the other orientations about this axis are), then its overall RT would also be long relative to that in Experiments 3 and 4 .

The fact that subjects had difficulty learning the shortest paths for orientations about at least some axes is inconsistent with the hypothesis that people spontaneously use shortest-path spatial transformations, as was argued by Shepard (1982, 1984). This observation is consistent with an analysis of the strengths and weaknesses of a shortest path procedure (Parsons, 1986a) and with my own unpublished studies of spatial reasoning.

\section{General Discussion}

\section{Properties of Imagined Spatial Transformations}

Imagined spatial transformations of different stimuli for a constant orientation difference. There are two possible causes of the pattern of discrimination and simulation RTs for different directions of OD. First, the RT patterns may be due to general properties of a subject's representation and imagined spatial transformation of shape. Second, the RT patterns may be due to the properties of the imagined object or of spatial transformations specifically associated with those objects.

In a companion study (Parsons, in press) that used the same planes of OD but abstract three-dimensional objects instead of body parts, RT-OD functions for the different planes were not correlated with those here. The relations among slopes and overall means for different planes of $O D$ were quite different. If imagined spatial transformations underlie discrimination functions for object and body stimuli, the differences in functions suggest that properties of imagined spatial transformations depend on the object imagined. If this is so, the properties observed here are not general properties of shape representation and imagined spatial transformations. Comparing the results of these two sets of studies is complicated by the fact that the two 
kinds of stimuli differ on more than a single dimension. The body is more familiar, it has familiar spatial transformations associated with it, and it has parts that may be spatially transformed relative to the whole. However, the preceding conclusion is reinforced by the comparison of the results here with those of studies of the left-right judgment and imagined spatial transformation of hands and feet. In those studies (Parsons, 1983b, 1987a), when ODs were comparable to those here, RTOD functions observed were dissimilar in shape and slope.

Evidence for the geometrical basis of imagined spatial transformations. The results in this article suggest that people spontaneously use quite efficient paths (e.g., spin-precession or shortest) for those orientations in Experiments 3 and 4. Such findings are consistent with those of other studies of imagined spatial transformations (Parsons, 1983b, 1987a, 1987b). In general, Parsons' results suggest that subjects imagine efficient (though not necessarily shortest) paths, though with some exceptions. In some cases, people appeared to use rotations by dimensions and other inefficient paths (as was indicated by Just \& Carpenter, 1985); and in a few cases, people seemed to use shortest paths. (Some of my unpublished findings indicate that people do not ordinarily know or use shortest paths between different orientations of an object if the spatial difference is not in one of the object's principal planes or in one of principal planes of the observer's visual frame of reference.) This variety of findings emphasizes that even though subjects appear to use efficient paths for a subset of ODs here (those in Experiment 5), they may use inefficient paths for other planes of OD. In general, it is possible that people use a variety of spatial transformations, depending on the properties of the stimulus and orientation difference. The variety of different strategies and procedures people use may also be influenced by properties of the experimental design and task, such as blocking trials by various factors. Further research is necessary to understand these details of human spatial transformations.

There is some evidence that imagined spatial transformations involving both translation (or size scaling) and reorientation are performed so that translation and reorientation occur sequentially but in small steps (Bundeson et al., 1983). Such a transformation is thought to produce an effectively homogeneous transformation in which the rate of translation and reorientation are proportional to the total translation (size scaling) and total reorientation required to complete the spatial transformation. This kind of model is consistent with the results and spatial transformation procedures discussed here. It is possible to couple any of the three reorientation procedures discussed here with a straight line translation "operator," because such reorientation and translation are orthogonal. A variant of any of the resulting three spatial transformation procedures would fit the Bundeson et al. results (cf. Parsons, 1986a).

Temporal and kinematic properties of imagined spatial transformations are strongly affected by properties of the imagined object. Overall, these findings (and, e.g., those in Parsons, $1983 \mathrm{~b}, 1987 \mathrm{a}, 1987 \mathrm{~b}$, in press) suggest that imagining an object's spatial transformation is not always a process of applying very general procedures, as could be assumed from earlier work (e.g., Shepard, 1975). The temporal and kinematic properties of imagined spatial transformations seem to be strongly influenced by properties of the imagined object. People do not seem to use spatial transformations that produce the same minimum-angle path with a uniform rate (and initiation time) for every object at every absolute orientation difference.

These interpretations raise the issue of how to compare the well-known "strong" model of imagined (mental) rotation, which has two parameters (rate and intercept), with other models (cf. Collyer, 1985). The more recent results are better fit by a "weaker" model which has several more parameters. For example, one parameter is the axis of rotation (i.e., time to plan shortest-path rotations for principal axes of an object or of observer's visual frame of reference may be shorter than that for other axes). Another factor is the interaction of axis and extent of rotation (i.e., rates vary for different directions). A third factor is the configuration or shape of the object (i.e., in the present case, whether the left or right arm is outstretched may influence rate or path of reorientation). In Experiments 3 and 4, a model adding all the ANOVA factors involving axis of orientation difference, to the "strong" model (that uses only orientation difference), accounts for 1.5 to 2 times the proportion of the total sums of squares accounted for by the "strong" model (the "weak" model accounts for 54\% and 23\% in Experiment 3 and 4 , the "strong" model, $35 \%$ and $12 \%$ ). Of course, models with more parameters usually fit better than those with fewer parameters because the additional parameters often capitalize on the error variance. The acceptance of these "weaker" models will depend on the usefulness and generality of their additional parameters.

\section{Imagined Spatial Transformations of One's Body and Perspective Taking}

The model of performance supported by the results in this article and in studies of left-right judgments of other body parts (Cooper \& Shepard, 1975; Parsons, 1983b, 1987a), suggests that the most convenient internal representation of body-part handedness for comparing with an external stimulus is that of one's own body. Furthermore, the most convenient spatial transformation to imagine is apparently that of imagining one's body part(s) at the orientation of the stimulus, rather than imagining the spatial transformation of the stimulus. This may be the most convenient spatial transformation to imagine if spatial transformation processes used in the planning (or execution) of one's actions are readily available for use in situations such as this task. This suggestion is consistent with the fact that in studies in which body-part stimuli were imagined, subjects report accompanying kinesthetic impressions or sensations (Cooper \& Shepard, 1975; Parsons, 1983b, Sekiyama, 1982). Such findings may lead to development of a model of imagined spatial transformations in which the underlying processes simulate or model concrete properties of an object's actual motion (e.g., its dynamic, kinematic, or kinesthetic properties).

It is interesting that the schematic line drawing of a human body, as was used in Experiments 1 and 2, can elicit spontaneous, rapid, and accurate imagined reorientations of one's self to the orientation of a stimulus. This is in contrast to results in several studies in which people are generally unable or unwilling to imagine a spatial transformation of their perspective or egocentric frame of reference (Cooper \& Shepard, 1973; Hintzman, O'Dell, \& Arndt, 1981; Huttenlocher \& Presson, 1973; 
Koriat \& Norman, 1984; Presson, 1982). They imagine instead the reorientation of external objects. Thus, for example, when reading a vertically held map, people often prefer to imagine or physically produce a spatial transformation of the map to a standard orientation, where the upward direction of the map is aligned with their forward direction in the environment (Levine, Jankovic, \& Palij, 1982; Shepard \& Hurwich, 1984). They do not imagine an egocentric frame (or their body) at different orientations along the route. Results of the kind presented here seem to have some obvious implications for the design of materials used in activities requiring spatial cognition.

The imagined spatial transformations studied here may not be identical to imagined spatial transformations of one's egocentric frame, although both transformations may allow one to know arbitrary directions at an arbitrary orientation. One approach to investigating this issue is to use a selective interference paradigm to see if performing a task that requires use of egocentric (and kinesthetic) information selectively disrupts concurrent use of imagined spatial transformations like those in this task.

A related possibility is that subjects in this task imagined the spatial transformation of (a) a canonical representation of their upright bodies rather than (b) their own bodies and accompanying egocentric frame. Such a hypothesis could be examined by assessing performance with the subject placed at different orientations with respect to the environment and stimulus. Corballis and his coworkers have investigated the effect of head tilt on discrimination and imagined rotation of letters, numbers, and dot patterns. Their results have been mixed (Corballis, Nagourney, Shetzer, \& Stefanatos, 1978): RT-OD functions shift in the direction of head tilt for judging the symmetry of dot patterns (Corballis \& Roldan, 1975; Corballis, Zbrodoff, \& Roldan, 1976) but not for discriminating correct and reversed letters and numbers (Corballis et al., 1976). Thus, different tasks may be differently affected by the subject's orientation with respect to the environment and stimuli.

The consequences of perceiving another human body and identifying one's orientation with its orientation can in some circumstances be distressing. Studies of space motion sickness (Oman, Lichtenberg, Money, \& McCoy, 1986) have observed that, in zero gravity, perceiving a person whose top-bottom orientation is opposite that to which one has adapted often rapidly induces the unpleasant symptoms of space motion sickness. Apparently, as the other person's orientation is recognized, there is a strong tendency to suddenly perceive oneself as being upside-down. Then, as one adjusts his or her understanding of the surroundings accordingly, there is a quick onset of the illness. Furthermore, the ability to imagine one's self at different orientations may be an important factor in the competent performance of airplane pilots (R. N. Shepard, personal communication, March 1986).

\section{References}

Bundeson, C., Larsen, A., \& Farrell, J. E. (1981). Mental transformations of size and orientation. In A. D. Baddeley \& I. B. Long (Eds.), Attention and performance $I X$ (pp. 279-294). Hillsdale, NJ: Erlbaum.

Collyer, C. E. (1985). Comparing strong and weak models by fitting them to computer-generated data. Perception \& Psychophysics. 38 , 476-481.

Cooper, L. A. (1975). Mental rotation of random two-dimensional shapes. Cogritive Psychology, 7, 20-43.

Cooper, L. A. (1976). Demonstration of a mental analog of an external rotation. Perception and Psychophysics, 19, 296-302.

Cooper, L. A., \& Shepard, R. N. (1973). Chronometric studies of the rotation of mental images. In W. G. Chase (Ed.), Visual information processing (pp. 75-176). New York: Academic Press.

Cooper, L. A., \& Shepard, R. N. (1975). Mental transformations in the identification of left and right hands. Journal of Experimental Psy chology: Human Perception and Performance, 104, 48-56.

Corballis, M. C., Nagourney, B., Shetzer, L. 1., Stefanatos, G. (1978). Mental rotation under head tilt: Factors influencing the location of the subject frame of reference. Perception and Psychophysics, 24, 263-272.

Corballis, M. C., \& Roldan, C. E. (1975). Detection of symmetry as a function of angular orientation. Journal of Experimental Psychology: Human Perception and Performance, 19, 221-230.

Corballis, M. C., Zbrodoff, N. J., \& Roldan, C. E (1976). What's up in mental rotation? Perception \& Psychophysics, 19, 525-530.

Corballis, M. C., Zbrodoff, J., Shetzer, L. I., \& Butler, P. B. (1978). Decisions about identity and orientation of rotated letters and digits. Memory \& Cognition, 6, 98-107.

Goldstein, H. (1950). Classical mechanics. Reading, MA: AddisonWesley.

Hinton, G. E., \& Parsons, L. M. (1981). Frames of reference and mental imagery. In A. D. Baddeley \& J. Long (Eds.), Attention and performance $I X$ (pp. 261-277). Hillsdale, NJ: Erlbaum.

Hinton, G. E., \& Parsons, L. M. (1987). Scene-based and viewer-related representations for comparing shapes. Manuscript submitted for publication.

Hintzman, D. L., O'Dell, C. S., \& Arndt, D. R. (1981). Orientation in cognitive maps. Cognitive Psychology, 13, 149-206.

Huttenlocher, J., \& Presson, C. C. (1973), Mental rotation and the perspective problem. Cognitive Psychology, 4, 277-299.

Just, M. A., \& Carpenter, P. A. (1985). Cognitive coordinate systems: accounts of mental rotation and individual differences in spatial ability. Psychological Review; $92,137-172$

Kaushall, P., \& Parsons, L. M. (1981). Optical information and practice in the discrimination of 3-D mirror-reflected objects. Perception. 10, 545-562.

Koriat, A., \& Norman, J. (1984). What is rotated in mental rotation? Journal of Experimental Psychology: Learning, Memory, and Cognition, $10,421-434$.

Levine, M., Jankovic, I. N., \& Palij, M. (1982). Principles of spatial problem solving. Journal of Experimental Psychology: General, $11 I_{*}$ 157-175.

Meztler, J. (1973). Cognitive analogues of the rotation of three-dimensional objects. Unpublished doctoral dissertation, Stanford University, Stanford, CA.

Metzler, J., \&hepard, R. N. (1974). Transformational studies of the internal representation of three-dimensional objects. In $\mathbf{R}$. Solso (Ed.), Theories in cognitive psychology: The Loyola Symposium (pp. 174-201). Hillsdale, NJ: Erlbaum.

Oman, C., Lichtenberg, B., Money, K., \& McCoy, R. (1986). Space motion sickness: Symptoms, stimuli, and predictability. Experimental Brain Research, 64, 316-334.

Parsons, L. M. (1983a). Imagined spatial transformations in the discrimination of left and right parts of the body. Proceedings of the Fifth Annual Conference of the Cognitive Science Society (pp. 47-51). Rochester, NY: Cognitive Science Society.

Parsons, L. M. (1983b). Mental rotation paths in the discrimination of 
left and right parts of the body. Unpublished doctoral dissertation, University of California, San Diego.

Parsons, L. M. (1986a). Evaluation and use of spatial transformation procedures in apparent motion and imagination. Manuscript submitted for publication.

Parsons, L. M. (1986b). Serial search and comparison of pairs of objects. Manuscript submitted for publication.

Parsons, L. M. (1986c). Use of a "body analogy" in the visual discrimination of abstract mirror-reflected shapes. Manuscript submitted for publication.

Parsons, L. M. (1987a). Imagined spatial transformation of one's hands and feet. Cognitive Psychology, 19, 178-241.

Parsons, L. M. (1987b). Imagined spatial transformations used in perception, imagination, and action. In L. Vaina (Ed.) Matters of intelligence (pp. 139-176). Dordrecht, the Netherlands: Reidel.

Parsons, L. M. (in press). Visual discrimination of abstract mirror-reflected three-dimensional objects. Perception and Psychophysics.

Parsons, L. M., \& Shimojo, S. (in press). Perceived spatial organization of cutaneous patterns on surfaces of the human body in various positions. Journal of Experimental Psychology: Human Perception and Performance.

Pinker, S. (1980). Mental imagery and the third dimension. Journal of Experimental Psychology: General, 109, 254-371.

Presson, C. C. (1982). Strategies in spatial reasoning. Journal of Experimental Psychology: Learning. Memory, and Cognition, 8, 243-251.
Ratcliff, G. (1979). Spatial thought, mental rotation, and the right cerebral hemisphere. Neuropsychologia, 17, 49-54.

Sayeki, Y. (1981). "Body analogy" and the cognition of rotated figures. The Quarterly Newsletter of the Laboratory of Comparative Human Cognition, 3, 36-40.

Sekiyama, K. (1982). Kinesthetic aspects of mental representations in the identification of left and right hands. Perception and Psychophysics, 32, 89-95.

Sekiyama, K. (1983). Mental and physical movements of hands: Kinesthetic information preserved in representational systems. Japanese Psychological Research, 25, 95-102.

Shepard, R. N. (1975). Form, formation, and transformation of internal representations. In R. Solso (Ed.), Information processing and cognition: The Loyola Symposium (pp. 87-117). Hillsdale, NJ: Erlbaum.

Shepard, R. N. (1982). Perceptual and analogical bases of cognition. In J. Mehler, M. Garrett, \& E. Walker (Eds.), Perspectives in mental representation (pp. 49-67). Hillsdale, NJ: Erlbaum.

Shepard, R. N. (1984). Ecological constraints on internal representation: Resonant kinematics of perceiving, imagining, thinking, and dreaming. Psychological Review, 91, 417-447.

Shepard, R. N., \& Hurwich, S. (1984). Spatial cognition, mental rotation, and interpretation of maps. Cognition, 18, 161-193.

Shepard, R. N., \& Metzler, J. (1971). Mental rotation of three-dimensional objects. Science, 191, 952-952.

Appendix

\section{Spatial Transformation Procedures}

This Appendix describes in more detail the three spatial transformation procedures discussed here (for a more formal treatment, see Parsons, 1986a). Each procedure requires a description of the orientation of a stimulus in direction cosines. Tables A-1 and A-2 show the degrees of rotation required for each of the three procedures to rotate the observer from his/her orientation to the orientation of the stimulus on each trial. These values are used to compare RTs in Experiments 1, 3, and 4 to these three spatial transformations.

\section{Rotations by Dimensions}

This procedure follows these steps. (a) Determine in which quadrant of space the final orientation of the major principal axis of the body is (e.g., forward, backward, leftward, and/or rightward). (b) Determine the angle from the final orientation of body's major principal axis to $\mathrm{YZ}$ and $\mathrm{XY}$ planes of the environmentally fixed frame with its origin at the body's center of mass (as in Figures 4-16). (c) Rotate the body in either $\mathrm{YZ}$ or $\mathrm{XY}$ plane (whichever is closer to the final orientation of the body's major principal axis). This rotation aligns the body's major principal axis with the projection of the vector representing its final orientation onto the plane it is being rotated in ( $Y Z$ or $X Y$ ). (d) If necessary, rotate the body about the vertical (i.e., Y) axis, so that the body's major principal axis is in its final orientations. Finally, (c) if necessary, spin the body about its major principal axis to put the minor and mean principal axes of the body at their final orientations.

A well-known example of a rotations-by-dimensions procedure is embodied in Euler angles, which are used to describe differences in orientation (cf. Goldstein, 1950). When the orientation difference is en-

Table A-1

Degrees of Rotation Between Subjects' Orientation During Task and Orientation of Stimulus for the Three Transformation Procedures: Experiments 1 and 2

\begin{tabular}{lccccccc}
\hline \multirow{2}{*}{$\begin{array}{c}\text { Orientation in } \\
\text { picture plane }\end{array}$} & 0 & 30 & 60 & 90 & 120 & 150 & 180 \\
\cline { 2 - 7 } & 0 & 30 & 60 & 90 & 120 & 150 & 180 \\
Back & 180 & 210 & 240 & 270 & 300 & 330 & 360 \\
Front & 180 & 182 & 190 & 201 & 216 & 234 & 254 \\
$\quad$ Rotations by dimensions & 180 & 180 & 180 & 180 & 180 & 180 \\
Spin precession & Shortest path & &
\end{tabular}

Note. This table does not include the angle of rotation required for reorientation of the arm. (This angle is approximately constant for all stimuli in these experiments.) 
Table A-2

Degrees of Rotation Between Subjects' Orientation During

Task and Orientation of Stimulus for the Three

Transformation Procedures: Experiments 3, 4, and 5

\begin{tabular}{lllllll}
\hline & \multicolumn{6}{c}{ Orientation difference } \\
\cline { 2 - 7 } Axis & 30 & 60 & 90 & 120 & 150 & 180 \\
\hline
\end{tabular}

\begin{tabular}{lrrrrrr}
\hline \multicolumn{7}{c}{ Rotations by dimensions } \\
$(1,0,0)$ & 30 & 60 & 90 & 120 & 150 & 180 \\
$(0,1,0)$ & 30 & 60 & 90 & 120 & 150 & 180 \\
$(0,0,1)$ & 30 & 60 & 90 & 120 & 150 & 180 \\
$(.71, .71,0)$ & 43 & 86 & 131 & 256 & 266 & 270 \\
$(0,71, .71)$ & 43 & 86 & 131 & 256 & 266 & 270 \\
$(.71,0, .71)$ & 120 & 150 & 180 & 210 & 240 & 270 \\
$(0,-.71, .71)$ & 43 & 86 & 131 & 256 & 266 & 270 \\
$(.71,0,-.71)$ & 120 & 150 & 180 & 210 & 240 & 270 \\
$(.71,-.71,0)$ & 43 & 86 & 131 & 256 & 266 & 270 \\
$(.58, .58, .58)$ & 114 & 138 & 161 & 180 & 234 & 290 \\
$(.58, .58,-.58)$ & 114 & 138 & 161 & 180 & 234 & 290 \\
$(.58,-.58, .58)$ & 114 & 138 & 161 & 180 & 234 & 290 \\
$(-.58, .58, .58)$ & 114 & 138 & 161 & 180 & 234 & 290 \\
\hline & & & & & &
\end{tabular}

Spin precession

\begin{tabular}{lllllll}
$(1,0,0)$ & 30 & 60 & 90 & 120 & 150 & 180 \\
$(0,1,0)$ & 30 & 60 & 90 & 120 & 150 & 180 \\
$(0,0,1)$ & 30 & 60 & 90 & 120 & 150 & 180 \\
$(.71, .71,0)$ & 30 & 61 & 93 & 127 & 163 & 201 \\
$(0, .71, .71)$ & 30 & 61 & 93 & 127 & 163 & 201 \\
$(.71,0, .71)$ & 30 & 60 & 90 & 120 & 150 & 180 \\
$(0,-.71, .71)$ & 30 & 61 & 93 & 127 & 163 & 201 \\
$(.71,0,-.71)$ & 30 & 60 & 90 & 120 & 150 & 180 \\
$(.71,-.71,0)$ & 30 & 61 & 93 & 127 & 163 & 201 \\
$(.58, .58, .58)$ & 30 & 61 & 93 & 127 & 163 & 211 \\
$(.58, .58,-.58)$ & 30 & 61 & 93 & 127 & 163 & 211 \\
$(.58,-.58, .58)$ & 30 & 61 & 93 & 127 & 163 & 211 \\
$(-.58, .58, .58)$ & 30 & 61 & 93 & 127 & 163 & 211 \\
\hline & & & & &
\end{tabular}

\begin{tabular}{lllllll} 
All axes & 30 & 60 & 90 & 120 & 150 & 180 \\
\hline
\end{tabular}

Note. This table does not include the angle of rotation required for reorientation of the arm. (This angle is approximately constant for all stimuli in these experiments.)

tirely in a principal plane of the observer's visual frame of reference, the rotations-by-dimensions procedure just described produces a path identical to that of the other procedures, assuming it uses principal axes of the observer's visual frame of reference. A more efficient version of this procedure evaluates how much orientation difference is eliminated by a rotation about each principal axis, and uses that rotation eliminating the most orientation difference overall. For some orientation differences, rotation about a príncipal axis will simultaneously eliminate ori- entation differences on two of three dimensions. A less efficient version of this procedure would randomly order the sequence of rotations about each principal axis on which there is an orientation difference.

\section{Spin Precession}

The spin-precession procedure discussed here spins the body about its major principal axis. It simultaneously rotates (precesses) it about the axis (in a transverse plane) that is the cross product of the vectors representing the initial and final orientations of the major principal axis. For simplicity, assume homogeneous motion, so that spin and precession rotations occur at coordinated rates proportional to the amount of rotation about each axis necessary to correct for the overall orientation difference. The precession angle is that between vectors representing initial and final orientations of the body's major principal axis. The spin angle is found by determining the amount of rotation necessary to align the minor and mean principal axes of the body once it has rotated through its precession angle.

The resulting spatial transformation is a rotation about an instantaneously changing axis. The total angle of rotation about this axis is equal to the precession angle plus the square root of the quantity 1 plus the square of the ratio of spin to precession angles (Parsons, 1986a). In principle, the spin can be about any axis, not just principal axes.

\section{Shortest Path}

The shortest-path procedure finds the axis (unique for each $O D$ ), about which the body can be rotated by an angle ( $180^{\circ}$ or less) that absolutely minimizes the degrees of rotation. The usual method for solving this problem (e.g., Goldstein, 1950) is as follows. (a) Describe one orientation in terms of the other, using direction cosines. (b) Construct the (change-of-basis) matrix $M$ by which the points described with respect to the initial reference frame can be described in terms of the final reference frame. (c) Set the major diagonal of this transformation matrix equal to 1 plus twice the $\operatorname{cosine}$ of $\theta$, and solve for $\theta$, the shortest path angle of rotation. (d) Find the eigenvector representing the axis of rotation.

Rotating the subject's body from its orientation in the task in Experiments 1 and 2 to the orientation portrayed by the front of the body in the picture plane (Figure 1) always requires $180^{\circ}$. This is because the change-of-basis matrix is

$$
M=\left[\begin{array}{rcc}
-1 & 0 & 0 \\
0 & -\sin \alpha & \cos \alpha \\
0 & \cos \alpha & \sin \alpha
\end{array}\right]
$$

where $\alpha$ is the angle of the body's major principal axis from upright. Thus,

$$
1+2 \cos \theta=-1-\sin \alpha+\sin \alpha .
$$

And $\cos \theta=-1$; or $\theta=180^{\circ}$, regardless of the value of $\alpha$, the picture plane orientation of stimulus.

Received July 14, 1986

Revision received December 2, 1986 